\title{
A Dynamic Model for the Efficiency Optimization of an Oscillatory Low Grade Heat Engine
}

\author{
Christos N. Markides ${ }^{1, *}$, Thomas C.B. Smith ${ }^{2}$ \\ ${ }^{I}$ Department of Chemical Engineering, Imperial College London, London SW7 2AZ, UK \\ ${ }^{2}$ Department of Engineering Science, University of Oxford, Oxford OX1 3PJ, UK
}

\begin{abstract}
A simple approach is presented for the modeling of complex oscillatory thermal-fluid systems capable of converting low grade heat into useful work. This approach is applied to the NIFTE, a novel low temperature difference heat utilization technology currently under development. Starting from a first-order linear dynamic model of the NIFTE that consists of a network of interconnected spatially lumped components, the effects of various component variables (geometric and other) on the thermodynamic efficiencies of the device are investigated parametrically. Critical components are highlighted that require careful design for the optimization of the device, namely the feedback valve, the power cylinder, the adiabatic volume and the thermal resistance in the heat exchangers. An efficient NIFTE design would feature a lower feedback valve resistance, with a shorter connection length and larger connection diameter; a smaller diameter but taller power cylinder; a larger (time-mean) combined vapor volume at the top part of the device; as well as improved heat transfer behavior (i.e. reduced thermal resistance) in the hot and cold heat exchanger blocks. These modifications have the potential of increasing the exergetic efficiency of the device by $50 \%$ points, corresponding to a $3.8 \%$ point increase in thermal efficiency.
\end{abstract}

Keywords: heat engine; thermofluidic oscillator; low grade heat; low temperature; linear model; efficiency

\section{INTRODUCTION}

With ever-increasing environmental concerns, including that of climate change, but also of energy security in the light of finite resources of common fossil fuels, it is becoming increasingly important to consider alternative clean and sustainable energy solutions. The efficiency and power density of appropriate thermodynamic systems are key quantities on which their usefulness and wider application rely. This is especially true for devices that are capable of operating with marginal energy sources and close to ambient temperatures. In this paper we present a modeling framework based on thermodynamic, heat transfer and fluid mechanical principles for the early stage development of oscillatory low grade heat (e.g. solar energy or waste heat) utilization systems. We demonstrate the approach by applying it to a promising new low grade heat utilization technology that has attracted attention recently as a result of potential reliability advantages as well as reduced capital and operating costs, on account of its few moving parts relative to mechanical heat engines. Specifically, we consider the effects of various system parameters on a number of key efficiency definitions that can be formulated in order to describe the performance of the system.

The "Non-Inertive-Feedback Thermofluidic Engine" (NIFTE), as proposed in Refs. [1-3], can be described as a "two-phase unsteady heat engine" in which persistent and reliable thermodynamic (pressure, temperature, etc.) oscillations are generated and sustained by steady external temperature differences. These desirable oscillations are driven by and give rise to heat and fluid flows, which involve the evaporation (boiling) and condensation of the working fluid. Hence, the NIFTE can also be considered a two-phase realization of a class of devices known as "thermofluidic oscillators", which includes thermoacoustic engines [4-7], dry free-liquidpiston (Fluidyne) engines [8-11], free-piston Stirling engines [12-14], pulsejets and pulse-tubes [15-19]. In common with many thermofluidic oscillators, the NIFTE is particularly well suited to the conversion of low grade heat. It can be used to produce useful (hydraulic) work for fluid pumping, heating and/or cooling and niche power generation applications; in a variety of solar thermal and energy recovery settings, and in combined heat and power-pumping (CHP) schemes. The NIFTE has been demonstrated as being capable of operating across temperature differences down to $30 \mathrm{~K}$ between the heat source and sink.

\footnotetext{
* Corresponding author. Telephone: +44 20759 41601. Email: c.markides@imperial.ac.uk
} 
Thermofluidic oscillators have many dynamic similarities with analogue electronic oscillator circuits, and thus, electrical analogies have been used to predict approximate stability/instability criteria and to estimate first order heat and work flows, and efficiencies. The analogies, pioneered by Backhaus and Swift [4], were extended to include a description of exergy (or availability) flows and to allow for exergy losses due to irreversible heat transfer in order to model the NIFTE by Smith [1]. This promising electrical analogy model showed good agreement with experimental trends obtained from measurements on an actual NIFTE prototype over a certain range of conditions and prototype design parameters, though it also showed non-negligible deviations in some cases [2,3]. Considerable effort is placed in these references to explain the underlying reasons for these deviations, including for example the existence of shuttle (irreversible heat transfer) loss mechanisms in non-heat exchanging (or, nominally adiabatic) components, and the presence of inertia in the real NIFTE. Unfortunately, the original model was not extended to include such effects, and specifically, although mentioned in this early work the effect of inertia was not studied in depth.

Yet, any such real system will encompass inertial effects to some extent due to the mass of any contained liquids. A dependence on inertia in order to allow the system sustained pressure oscillations with large amplitudes, which is vital for the achievement of high power densities, implies a requirement for bulky tuning lines and resonators that the designer would like to avoid. An understanding of the need for and of the effects of finite inertia is crucial for the design of the next generation of these novel and complex systems, both in terms of enhanced efficiency and performance. The effects of inertia were incorporated into the original "non-inertive" electrical analogy model of Smith [1], by Solanki et al. [20]. The current work uses this latter "inertive" model as a starting point.

In Solanki et al. [20] the authors studied the effect of introducing (and parametrically varying the degree of) inertia on key NIFTE performance indicators, such as the minimum temperature of the supplied heat necessary for operation (sustained oscillations) and the resulting oscillation frequency. The revised inertive model resulted in a much improved prediction of these indicators relative to the original non-inertive equivalent, over a wider range of values. This suggests that the inclusion of inertia is indeed essential for the correct capture of the observed behavior of the NIFTE. The inertive model was then used to investigate the expected efficiency of the NIFTE.

However, the study focused only on the exergetic (second law) efficiency of the engine and did not present results for its thermal (first law) efficiency. In addition we note that, in general, the real NIFTE is an energy producing device that can have a different (indeed, reduced) global efficiency from the efficiency of its enclosed thermodynamic cycle, owing to processes of parasitic dissipation that are external to the working fluid cycle, but still internal to the overall device. In fact, the NIFTE depends on a parasitic throttle valve (referred to as the "feedback valve") to create a phase shift between the heat flow and the power stroke. The existence of this phase shift results in a parasitic viscous loss that leads to a difference between the efficiency of the thermodynamic cycle undergone by the working fluid, and the efficiency of the overall device. Therefore one needs to distinguish between four measures of efficiency: thermal and exergetic overall/global device efficiencies; and thermal and exergetic working fluid efficiencies. Solanki et al. [20] restricted their attention to the exergetic overall device efficiency, while in Refs. [1-3] the parasitic loses were assumed to be negligible altogether (i.e. no distinction was made between device and system efficiencies). In this paper, we investigate the consequences of this assumption and show that it is not valid in certain regions of the parameter space. We use this result to construct a framework for including parasitic losses in future thermofluidic oscillator models, by considering all four thermodynamic efficiency definitions in the inertive model to gain a more complete understanding of the thermodynamic performance potential of the NIFTE.

\section{METHODS}

\subsection{Problem Formulation: Heat, Power, Exergy, and Efficiency Definitions}

Consider an arbitrary, externally heated work-producing device, within which power is generated by a working fluid that undergoes a thermodynamic cycle. Rather than dealing with thermodynamic states, we will deal with time-averaged flow rates of thermodynamic properties (e.g. "entropy flow rate" $\dot{S}$ ), so that irreversible processes may be quantified legitimately as increases in entropy flow rate. Lower case letters are used to indicate specific quantities (per unit mass of working fluid), such that for instance, $\dot{s}$ is the specific entropy flow rate.

The temperature of the working fluid of the device that undergoes this "main cycle" can be written as a decomposition $T=T_{\mathrm{o}}+T^{\prime}$, where $T^{\prime}$ are fluctuations about the time-averaged value $T_{\mathrm{o}}$. By definition the timeaverage of the fluctuations is zero $\overline{T^{\prime}}=0$. The working fluid is heated by an external source and cooled by an external sink, through contact with appropriate heat exchangers. The source and sink on the other side of the heat exchangers undergo separate processes. Let us denote by $T_{\mathrm{h}}$ the temperature of the source from which the main 
cycle obtains heat, and by $T_{\mathrm{c}}$ the temperature of the sink to which it rejects heat. Assuming that the main cycle is heated and cooled by purely irreversible heat transfer, i.e. conduction, $T_{\mathrm{h}}$ and $T_{\mathrm{c}}$ are bounded by lines of constant heat flow $\dot{Q}$ that set a limit to the extent of the main cycle, wherein $T=\dot{Q} / \dot{S}$. In addition, let $\Delta \dot{S}$ be the entropy change during the processes of heat addition and rejection that are equal in magnitude, but opposite in sign; and let $V$ be the volume of the working fluid, such that $U=\dot{V}$ is the volumetric displacement flow rate responsible for power generation. Then, based on an arbitrary datum state (e.g. chose the time-averaged $T_{\mathrm{o}}, P_{\mathrm{o}}$ ), the rate of heat input to the main cycle, the net power produced, and the "net exergy (or equivalently availability) flow rate" are,

$$
\begin{gathered}
\dot{Q}_{\text {in }}=\int_{\Delta \dot{\mathrm{S}}>0} T(\dot{S}) \mathrm{d} \dot{S}=T_{\mathrm{o}} \Delta \dot{S}+\int_{\Delta \dot{\mathrm{S}}>0} T^{\prime}(\dot{S}) \mathrm{d} \dot{S}, \\
\dot{W}=\oint P(\dot{V}) \mathrm{d} \dot{V}=\oint P(U) \mathrm{d} U=\dot{Q}=\oint T(\dot{S}) \mathrm{d} \dot{S}, \\
\dot{X}_{\text {in }}=\int_{\Delta \dot{\mathrm{S}}>0}\left[T_{\mathrm{h}}(\dot{S})-T_{\mathrm{o}}\right] \mathrm{d} \dot{S} ; \dot{X}_{\text {out }}=\int_{\Delta \dot{\mathrm{s}}<0}\left[T_{\mathrm{c}}(\dot{S})-T_{\mathrm{o}}\right] \mathrm{d} \dot{S} ; \dot{X}=\dot{X}_{\text {in }}-\dot{X}_{\text {out }}=\oint T_{\mathrm{s}}(\dot{S}) \mathrm{d} \dot{S},
\end{gathered}
$$

where $T_{\mathrm{S}}$ is used to denote either $T_{\mathrm{h}}$ or $T_{\mathrm{c}}$ during the sequential heating $(\Delta \dot{S}>0)$ and cooling $(\Delta \dot{S}<0)$ parts of the cycle. From Eq. (1) we can see that the power produced by the device $\dot{W}=\dot{Q}$ may be described by the area inside a $T-\dot{S}$ diagram that represents the internally reversible part of the main thermodynamic cycle. This area is enclosed by a larger area that represents the net exergy flow rate $\dot{X}$ of the full irreversible cycle, with the difference between these areas representing power loss through irreversible processes. Hence, $\dot{X}$ is the maximum power available to the cycle given the boundary conditions. It is clear from Eqs. (2) and (3) that changes in $T_{\mathrm{o}}$ will have no effect on $\dot{W}$ or $\dot{X}$. However, from Eq. (1), we observe that $\dot{Q}_{\text {in }}$ will depend on $T_{\mathrm{o}}$, and in fact on the magnitude of $T^{\prime}$ relative to $T_{\mathrm{o}}$.

Next we consider two classes of efficiency: (1) the thermal (first law) efficiency $\eta_{\mathrm{th}}$, defined as $\eta_{\mathrm{th}}=\dot{W} / \dot{Q}_{\text {in }}$; and (2) the exergetic (second law) efficiency $\eta_{\mathrm{ex}}$, defined as $\eta_{\mathrm{ex}}=\dot{W} / \dot{X}$. In earlier models of the NIFTE, viscous dissipation in parasitic components was assumed negligible [1-3]. We refer to efficiencies based on this assumption as (thermal or exergetic) "working fluid" efficiencies $\eta_{\mathrm{wfl}}$ and efficiencies for which parasitic dissipation is accounted for as (thermal or exergetic) "device" efficiencies $\eta_{\mathrm{dev}}$. In this paper we consider the effect of various device parameters on these efficiencies, with reference to the NIFTE which is introduced in more detail below.

\subsection{Non-Inertive-Feedback Thermofluidic Engine (NIFTE)}

A schematic of the NIFTE can be seen in Fig. 1. Details of the construction and operation of the NIFTE can be found elsewhere [3], but briefly the NIFTE comprises two vertical cylinders $(1,2)$, connected at the top (3) and bottom (4) with horizontal pipes. The whole interconnected volume contained within these main components (chambers and tubes) is filled with a single working fluid that exists in both the vapor (white) and the liquid (gray) phase at the same time. The liquid and vapor volumes are separated by two vapor-liquid interfaces denoted in Fig. 1 by levels (6) and (7), the first of which is inside the power cylinder on the left (1) and the second is inside the displacer cylinder on the right (2). The total volume of vapor at the top of the NIFTE (8) includes the vapor volumes in the power and displacer cylinders, together with the volume of the horizontal "vapor connection" pipe (3). This combined space is assumed to be an "adiabatic vapor chamber" (8). The second horizontal pipe that connects the power and displacer cylinders further down is referred to as the "liquid feedback connection" (4) and contains a valve termed the "feedback valve" (5). The power cylinder (1) transmits the fluid displacement to the load (9) below it. We treat the case where the working fluid and power transmitting fluid are the same.

The displacer cylinder (2) contains two heat exchanger blocks arranged vertically, namely the hot (10) and cold (11) heat exchangers. The heat transfer coefficient associated with single phase convection is much lower (typically by orders of magnitude) than that associated with phase change, and hence we neglect all modes of heat transfer other than evaporation and condensation. Evaporation occurs when working fluid in the liquid phase comes into contact with the surface of the hot heat exchanger (10), whereas condensation occurs when working fluid in the vapor phase comes into contact with the cold heat exchanger (11). The latter is the dominant heat transfer process at the instant portrayed in Fig. 1, when the vapor-liquid interface (7) is at the height of the cold heat exchanger (11).

Starting from this instant of condensation, the reduction of volume/mass of working fluid in the vapor phase (white) leads to a decrease in pressure in the vapor volume of the NIFTE (i.e. the combined space at the top of the power and displacer cylinders (8), and in the vapor connection). This reduction in pressure causes level 6 to be forced upwards in the power cylinder (1), and fluid to be sucked into the NIFTE from the load line (9). As level 6 rises in the power cylinder (1), the hydrostatic pressure difference between the power (1) and displacer (2) cylinders first decelerates and then reverses the upwards motion of level 6 . The reduced pressure also causes level 7 to rise, bringing liquid into contact with the hot heat exchanger (10). The evaporation of this liquid increases of 
volume of vapor (white) and leads to pressurization of the vapor volume that forces level 6 downwards, while fluid is displaced out of the NIFTE into the load line (9). As level 6 falls, the hydrostatic pressure difference between the power (1) and displacer (2) cylinders first decelerates and then reverses the downwards motion of level 6. The pressurization also causes level 7 to descend onto the cold heat exchanger whence condensation can re-commence, completing one cycle of the NIFTE. The process then repeats itself. The oscillating fluid displacement into and out of the load line (9) can be harnessed in various ways, one of which involves the use of a pair of check valves in opposite directions and in parallel to each other in order to achieve a uni-directional flow for pumping [3]. A linear approximation of the operation of the NIFTE can be described by an LRC circuit, that is, an electrical circuit with inductors $(L)$, resistors $(R)$ and capacitors $(C)$, as explained in the following section.

\subsection{Spatially Lumped and One-Dimensional Linear Dynamic Model}

In Smith [1-3] a simple, but powerful model for the dynamic behavior of the NIFTE was proposed, following Backhaus and Swift [4], Ceperley [7] and Huang and Chuang [15]. This involved lumped (spatially averaged, and thus independent) and one-dimensional linearized sub-models for each NIFTE component that were interconnected to derive a complete model for the whole device. The linearization allows analogies to be drawn with analogue electrical components, thus enabling an electrical network to be constructed for the NIFTE. The network contains resistors (accounting for viscosity, fluid drag and thermal resistance), and capacitors (accounting for gravity and compressibility). Here we extend this approach, with the added inclusion of inductors to capture finite inertial effects, in line with Solanki et al. [20].

In more detail, the dominant physical process undergone in each sub-component is firstly identified and modeled linearly. By drawing analogies between the physical variables of pressure $(P)$ and voltage $(E)$, volumetric flow rate $(U)$ and current $(I)$, temperature $(T)$ and voltage $(E)$, and entropy flow rate $(\dot{S})$ and current $(I)$, the linear, lumped equations involving the raw thermal-fluid variables are transformed into a suitable network of electrical elements.

Referring to the electrical network in Fig. 2, resistances to heat or fluid flow are represented by a resistors $(R)$; hydrostatic pressure (i.e. gravitational potential energy) of the liquid and adiabatic compressibility of the vapor are both represented by capacitors $(C)$; and fluid inertia is represented by inductors $(L)$. The dynamic equation of each element is $I=E / R, I=C \cdot \dot{E}$ and $\dot{I}=E / L$, respectively, where $E$ is the potential difference across the component and $I$ is the current through it. The physical quantities used to evaluate the value of each electrical component in Fig. 2 are shown in Table 1, with an explanation of all variables in the caption.

Note that the network in Fig. 2 represents a model of connected interacting processes, and that some of these are thermal (heat) flow processes while others are fluid flow processes. So, for example, consider the adiabatic compression/expansion process undergone by the working fluid vapor in the vapor chamber ( 8 in Fig. 1) due to the time-varying changes to the liquid levels in the power (6) and displacer (7) cylinders. This is a 'fluid' process and as such can be purely described by changes in a pressure $(P)$ and a volumetric flow rate $(U)$. In this case, pressure is the 'potential' (or 'voltage') and the volumetric flow rate is the 'current'. Let $P_{\mathrm{ad}}, V_{\mathrm{ad}}$ be the time-varying pressure and volume of the vapor, such that the process can be described by $P_{\mathrm{ad}} V_{\mathrm{ad}}^{\gamma}=$ const. For small perturbations of pressure and volume around an equilibrium $P_{\mathrm{ao}}, V_{\mathrm{ao}}$ we have,

$$
\frac{1}{P_{\mathrm{ao}}} \frac{\mathrm{d} P_{\mathrm{ad}}}{\mathrm{d} t}+\frac{\gamma}{V_{\mathrm{ao}}} \frac{\mathrm{d} V_{\mathrm{ad}}}{\mathrm{d} t}=0 \Longrightarrow U_{\mathrm{ad}}(t)=\frac{V_{\mathrm{ao}}}{\gamma P_{\mathrm{ao}}} \frac{\mathrm{d} P_{\mathrm{ad}}(t)}{\mathrm{d} t}=\frac{V_{\mathrm{ao}}}{\gamma P_{\mathrm{ao}}} \dot{P}_{\mathrm{ad}}(t) \text { or } u_{\mathrm{ad}}(\tilde{S})=\frac{V_{\mathrm{ao}}}{\gamma P_{\mathrm{ao}}} \tilde{s} P_{\mathrm{ad}}(\tilde{s})
$$

that behaves equivalently to a capacitor $I=C_{\mathrm{ad}} . \dot{E}$, with $C_{\mathrm{ad}}=V_{\mathrm{ao}} / \gamma P_{\mathrm{ao}}$. Note that here, $\tilde{s}$ is the Laplace operator.

In another example, we assume that the fluid flow in the load (9) is akin to viscous laminar flow in a smooth pipe (i.e. at low Reynolds numbers $R e$ ) and at low frequencies (and hence also low Womersley parameters $W o$ ) when the flow is quasi-steady. Let $P_{1}$ be the pressure difference across the load and $U_{1}$ the volumetric flow rate through it. In the absence of inertia ( $L_{1}=0$ in Fig. 2) $P_{1}$ acts only across $R_{1}$, and the flow resistance (i.e. drag $D$ ) is modeled as,

$$
D=\frac{P_{1}}{\frac{1}{2} \rho u_{1}^{2}} \frac{l_{1}}{d_{1}}=\frac{64}{R e_{\mathrm{d}}} \Rightarrow U_{\mathrm{l}}=\frac{1}{128 \mu l_{\mathrm{l}} / \pi d_{\mathrm{l}}{ }^{4}} P_{\mathrm{l}},
$$

that resembles a resistor $I=1 / R_{1} . E$, with $R_{1}=128 \mu l_{1} / \pi d_{1}{ }^{4}$. Here, $u_{1}=U_{1} /\left(\pi d_{1}^{2} / 4\right)$ is the bulk velocity in the load. At higher frequencies one can no longer neglect inertia, which is then included in the form of an inductor $L_{1}$ in Fig. 2. This component is sufficient to account for changes in the amplitude and the phase of $P_{1}$ relative to $U_{1}$ [21].

Now, consider instead the section indicated by the enclosed box in Fig. 2. This represents the heat exchangers (10 and 11), and specifically, the process of two-phase heat transfer by which the periodic lapping of the hot/cold heat 
exchanger blocks by the working fluid liquid level (7) in the displacer cylinder (2) drives an alternating process of generation/condensation of vapor directly into/out of the adiabatic vapor chamber (8). This is a 'thermal' process that can be described by changes in temperature $(T)$ and entropy flow rate $(\dot{S})$, whereby the 'potential' (or 'voltage') is now the temperature and the 'current' is the entropy flow rate. This process is described by,

$$
\dot{S}_{\text {th }}=\frac{1}{T_{\mathrm{o}} / k}\left(T_{\mathrm{s}}-T\right) \text {, }
$$

where $k$ is the thermal conductance (inverse of the thermal resistance) between the solid block and the working fluid, and $T_{\mathrm{o}}$ is the mean boiling point temperature over which the working fluid evaporates and condenses. For the purposes of our model, $T_{\mathrm{o}}$ is the saturation temperature that corresponds to a saturated vapor pressure of the working fluid equal to the time-averaged pressure in the adiabatic vapor chamber $\overline{P_{\mathrm{ad}}}$. It is also equal to the mean temperature between the hot source and cold sink, as well as the time-averaged working fluid temperature in the vapor phase within the adiabatic vapor chamber $T_{\mathrm{o}}=\overline{T_{\mathrm{S}}}=\bar{T}$.

Returning to the section indicated by the enclosed box in Fig. 2, we note that the thermal process variables must be coupled to (or referred to) the fluid variables in the rest of the circuit. For this, we use a pair of coupling equations, one for the potential variable,

$$
T_{\mathrm{s}}=\left(\frac{\partial T}{\partial P}\right)_{\mathrm{fg}} P_{\mathrm{th}} ; T=\left(\frac{\partial T}{\partial P}\right)_{\mathrm{fg}} P_{\mathrm{ad}},
$$

and one for the flow variable,

$$
\dot{S}_{\mathrm{th}}=\dot{m}_{\mathrm{th}} \Delta s_{\mathrm{fg}}=\frac{\Delta s_{\mathrm{fg}}}{\Delta v_{\mathrm{fg}}} U_{\mathrm{th}},
$$

such that Eq. (6), which describes the process of two-phase heat transfer in the heat exchangers, can be re-written as,

$$
U_{\mathrm{th}}=\frac{\left(\frac{\partial T}{\partial P}\right)_{\mathrm{fg}}}{\frac{\Delta S_{\mathrm{fg}}}{\Delta v_{\mathrm{fg}}} T_{\mathrm{o}} / k}\left(P_{\mathrm{th}}-P_{\mathrm{ad}}\right)=\frac{1}{\left(\Delta \Delta_{\mathrm{fg}} / \Delta v_{\mathrm{fg}}\right)^{2} T_{\mathrm{o}} / k}\left(P_{\mathrm{th}}-P_{\mathrm{ad}}\right)
$$

This behavior can be modeled by resistor $I=1 / R_{\mathrm{th}} . E$, with $R_{\mathrm{th}}=\left(\Delta s_{\mathrm{fg}} / \Delta v_{\mathrm{fg}}\right)^{2} T_{\mathrm{o}} / k$.

Results concerning all components in Fig. 2 are summarised in Table 1. Finally, it should be stated that the choice of all thermal-fluid-electrical analogies has been made to enforce the condition that electrical power, which can only be dissipated in resistors, is directly equal to a rate of heat transferred in the thermal domain $I . E=U . \Delta P=\dot{S} . \Delta T=$ $\dot{Q}$ or to a rate of work done (or lost is the component is parasitic) in the fluid domain $I . E=U . \Delta P$.

\subsection{Solution Procedure}

Similarly to Ref. [20], a "nominal" value of each electrical parameter in Fig. 2 was calculated in the first instance based on the expressions in Table 1. The nominal value was obtained from the known geometric design variables, the solid properties of the selected construction materials, and the fluid properties of the selected working fluid from the actual NIFTE prototype described in detail in Ref. [3]. From this combination of nominal parameters, we proceeded to perform a parametric investigation in which each $R, C$ and $L$ parameter was varied independently inside a certain range, while the rest were kept at their nominal value. The ranges for each parameter were selected so as to describe a reasonable incremental design perturbation from the existing NIFTE prototype, always leading to an acceptable and realistic configuration that we deem as practically achievable in the short term. Table 2 shows the nominal values and investigated ranges of each parameter.

Since all aforementioned components are assumed linear, the complete NIFTE network is also linear. For simplicity, but without loss of generality, we treat the case of sinusoidal oscillations with a single angular frequency $\omega$ in all quantities. We limit ourselves to considering the (efficiency) performance of the NIFTE at marginal stability, i.e. in conditions in which it exhibits sustained oscillations of constant amplitude with minimal gain. The internal feedback process with gain $G$, which can be seen on the far left in Fig. 2, is increased until this marginal stability condition is established. The gain is related directly to the spatial temperature gradient established in the source/sink heat exchanger configuration, and thus the difference between the hot and cold temperatures available externally to the device and the design of the heat exchanger configuration. In a real NIFTE a gain setting higher than marginal 
stability will not result in an unstable system, as the system will not be able to sustain continually increasing oscillations and will eventually saturate in a complex non-linear limit cycle behavior. However, this is a sub-optimal setting from the point of view that it demands a higher heat input temperature than that at the marginal stability condition. Thus, the marginal stability set-point can be interpreted as the lowest heat input temperature condition for which a given NIFTE configuration will achieve continuous oscillations.

At this point we note the relative amplitudes of oscillation of all variables. These are then used in order to evaluate the integrals for heat input $\dot{Q}_{\text {in }}$, net power $\dot{W}$ and net exergy $\dot{X}$ in Eqs. (1), (2) and (3), which for single-frequency sinusoidal signals simplify to,

$$
\begin{gathered}
\dot{Q}_{\text {in }}=T_{\mathrm{o}} \Delta \dot{S}+\omega \widehat{T}_{\mathrm{s}} \hat{S} \cos \measuredangle\left\{T_{\mathrm{s}}, \dot{S}\right\} / 4 \\
\dot{W}=\hat{P} \widehat{U} \cos \measuredangle\{P, U\} / 2 \\
\dot{X}=\omega \widehat{T}_{\mathrm{s}} \hat{S} \cos \measuredangle\left\{T_{\mathrm{s}}, \dot{S}\right\} / 2
\end{gathered}
$$

where $\widehat{T}$ and $\widehat{T}_{\mathrm{S}}$ are the temperature amplitudes of the main cycle (i.e. the working fluid) and its thermal environment (measured at the internal surface of the source/sink heat exchangers) respectively, $\hat{S}=\pi \Delta \dot{S} / \omega$ is the entropy flow amplitude of the main cycle, and $\measuredangle\{a, b\}$ is the phase angle between variables $a$ and $b$. Finally, from the quantities in Eqs. (10), (11) and (12) we can evaluate all required efficiencies defined previously. Hence, for the thermal efficiency of the working fluid $\eta_{\text {th,wfl }}$ we obtain the expression,

$$
\eta_{\text {th,wfl }}=\frac{\dot{W}_{\mathrm{wfl}}}{\dot{Q}_{\text {in }}}=2 \pi \frac{\hat{P}_{\mathrm{ad}}}{\hat{P}_{\mathrm{th}}} \cos \measuredangle\left\{P_{\mathrm{ad}}, U_{\mathrm{th}}\right\}\left(\frac{4}{\hat{T}_{\mathrm{s}} / T_{\mathrm{o}}}+\pi \cos \measuredangle\left\{T_{\mathrm{s}}, \dot{S}\right\}\right)^{-1},
$$

while for the thermal efficiency of the overall device $\eta_{\mathrm{th}, \mathrm{dev}}$ we have,

$$
\eta_{\text {th,dev }}=\frac{\dot{W}_{\text {dev }}}{\dot{Q}_{\text {in }}}=2 \pi \frac{\hat{P}_{1}}{\hat{P}_{\text {th }}} \frac{\widehat{U}_{1}}{\widehat{U}_{\text {th }}} \cos \measuredangle\left\{P_{1}, U_{1}\right\}\left(\frac{4}{\widehat{T}_{\mathrm{s}} / T_{\mathrm{o}}}+\pi \cos \measuredangle\left\{T_{\mathrm{s}}, \dot{S}\right\}\right)^{-1} .
$$

For the two thermal efficiencies $\eta_{\text {th }}$ we have used $\widehat{T}_{\mathrm{s}} / T_{\mathrm{o}}=0.05$ and $\overline{T_{\mathrm{s}}}=T_{\mathrm{o}}$, i.e. a $30 \mathrm{~K}$ peak difference between the hot source and cold sink at $T_{\mathrm{o}}=300 \mathrm{~K}$. The maximum Carnot efficiency, based on hot and cold temperatures of 315 and $285 \mathrm{~K}$ respectively, is $9.5 \%$.

\section{RESULTS AND DISCUSSION}

This section considers the thermal efficiency of the NIFTE, defined as $\eta_{\text {th }}=\dot{W} / \dot{Q}_{\text {in }}$, and the exergetic efficiency of the NIFTE, defined as $\eta_{\mathrm{ex}}=\dot{W} / \dot{X}$, which were evaluated from the expressions in Eqns. 10 to 14 . Efficiencies based on the assumption that viscous dissipation in parasitic components is negligible (as in Refs. [1-3]) are referred to as working fluid efficiencies $\eta_{\mathrm{wfl}}$ and describe the efficiency of the thermodynamic cycle undergone by the working fluid; whereas efficiencies which account for parasitic dissipation are referred to as device efficiencies $\eta_{\mathrm{dev}}$ and describe the efficiency of the overall device. Based on these definitions, results are presented in Figs. 3-8 for the effects of selected design parameters $(R \mathrm{~s}, C \mathrm{~s}, L \mathrm{~s}$ in Fig. 2 and Table 1$)$ on the NIFTE efficiencies:

(i) Thermal efficiency of the device $\eta_{\text {th,dev }}$;

(ii) Thermal efficiency of the working fluid system $\eta_{\text {th,wfl }}$;

(iii) Exergetic efficiency of the device $\eta_{\text {ex,dev }}$; and,

(iv) Exergetic efficiency of the working fluid $\eta_{\text {ex,wfl }}$.

Specifically, Fig. 3 shows results relating to the design of the power cylinder (component 1 in Fig. 2), Fig. 4 shows results relating to the design of the displacer cylinder (2 in Fig. 2), Fig. 5 shows results relating to the design of the feedback connection ( 4 and 5 in Fig. 2), and Fig. 6 shows results relating to the design of the heat exchangers (10 and 11 in Fig. 2) and the adiabatic vapor chamber (the total white region 8 in Fig. 2). The abscissas (on the horizontal axis) are normalized by the largest value in the investigated range. All efficiency values obtained from perturbations of the NIFTE from a nominal design can be compared to the efficiencies of the nominal NIFTE (i.e. corresponding to the actual NIFTE prototype described in Ref. [3]), which are summarized in Table 3.

By means of comparison, estimated values of $\eta_{\mathrm{ex}, \mathrm{dev}}$ from experiments on a NIFTE water pump [3] with a 150 W heater sustaining a heater temperature of $90{ }^{\circ} \mathrm{C}$ and a cooling water temperature of $4{ }^{\circ} \mathrm{C}$ were in the range $0.4-$ $1.7 \%$, while $\eta_{\text {ex,wfl }}$ was between 3.7 and $9.5 \%$. Based on these source and sink temperatures the Carnot efficiency is 
$24 \%$, which corresponds to $\eta_{\mathrm{th}, \mathrm{dev}}=0.1-0.4 \%$ and $\eta_{\mathrm{th}, \mathrm{wfl}}=0.9-2.3 \%$. Even though these reported ranges cover configurations that extend beyond nominal settings, they provide some confidence that the values provided in Table 3 are consistent with realistic NIFTE performance characteristics and a valid starting point for this current analysis.

Finally, Figs. 7 and 8 contain results of a sensitivity analysis that was performed in order to identify the key parameters that control the efficiencies of the NIFTE. In all plots, the parameter under investigation was varied independently, with all other parameters set to a nominal value. Here, the abscissa values of impedance on the horizontal axes have been normalized by each variable's nominal value.

\subsection{Optimal Design of the Power Cylinder}

Variations in the thermal efficiency $\eta_{\text {th }}$ (left) and exergetic efficiency $\eta_{\text {ex }}$ (right) due to changes in the design of the power cylinder are shown in Fig. 3. The maximum achievable efficiency values obtained by making these changes are $\eta_{\mathrm{th}}=5.6 \%$ and $\eta_{\mathrm{ex}}=76 \%$. These are considerably higher than the values stated in Table 3 for nominal NIFTE design and suggest that step improvements are possible. Recall that the capacitance and inductance of the power cylinder are given by the expressions $C_{\mathrm{p}}=\pi d_{\mathrm{p}}^{2} / 4 \rho g$ and $L_{\mathrm{p}}=4 \rho l_{\mathrm{p}} / \pi d_{\mathrm{p}}^{2}$, respectively. Hence:

- An increase in the diameter of the power cylinder $d_{\mathrm{p}}$ would cause $C_{\mathrm{p}}$ to increase and $L_{\mathrm{p}}$ to decrease;

- An increase in the liquid density of the working fluid $\rho$ would cause $C_{\mathrm{p}}$ to decrease and $L_{\mathrm{p}}$ to increase; and,

- An increase in the time-averaged length of the liquid column in the power cylinder $l_{\mathrm{p}}$ (related to the time-average of level 6 in Fig. 2) would cause $L_{\mathrm{p}}$ to increase, but would have no effect on $C_{\mathrm{p}}$.

In Fig. 3 we note that the two types of device efficiency (thermal and exergetic) lead to consistent design interpretations. For example, both $\eta_{\mathrm{th}}$ and $\eta_{\mathrm{ex}}$ experience a sharp drop as $L_{\mathrm{p}}$ is reduced below 0.3 , and a sharp rise as $C_{\mathrm{p}}$ is reduced below 0.2. This similarity can be also observed Figs. 4, 5 and 6 that relate to the displacer cylinder, feedback valve, heat exchangers and adiabatic vapor volume. However, the device efficiency is not necessarily optimal when the working fluid cycle exhibits its maximum efficiency. For example, looking at the figure on the left, $\eta_{\mathrm{dev}}$ is relatively insensitive to changes in $C_{\mathrm{p}}$ except at extremely low values (lower than about 0.05 ) where it shows an improvement, while $\eta_{\text {wfl }}$ increases considerably as $C_{\mathrm{p}}$ is gradually reduced below 0.2 . This contradiction will be even more evident in Fig. 4 that relates to the effect of the displacer cylinder.

From Fig. 3 it can be seen that $C_{\mathrm{p}}$ and $L_{\mathrm{p}}$ can be used to make significant improvements to the two device efficiencies, $\eta_{\text {th,dev }}$ and $\eta_{\text {ex,dev }}$. In absolute terms, $C_{\mathrm{p}}$ can be used to increase $\eta_{\text {th,dev }}$ by up to $2.0 \%$ and $\eta_{\text {ex,dev }}$ by up to $26 \%$, while $L_{\mathrm{p}}$ can be used to increase $\eta_{\mathrm{th} \text {,dev }}$ by up to $4.4 \%$ and $\eta_{\mathrm{ex} \text {,dev }}$ by up to $61 \%$. From this observation it becomes evident that the power cylinder (i.e. the choice of $C_{\mathrm{p}}$ and $L_{\mathrm{p}}$ ) is an important component in determining the overall efficiency of the NIFTE. Specifically, improved NIFTE efficiencies can be achieved by reducing $C_{\mathrm{p}}$ and increasing $L_{\mathrm{p}}$. Both of these improvements can be realized simultaneously by decreasing the diameter of the power cylinder $d_{\mathrm{p}}$ and increasing the liquid density of the working fluid $\rho$. An additional increase in the length of the liquid column in the power cylinder $l_{\mathrm{p}}$ would act to further increase $L_{\mathrm{p}}$, without affecting $C_{\mathrm{p}}$.

Changes to $C_{\mathrm{p}}$ and $L_{\mathrm{p}}$ can also considerably affect the two working fluid efficiencies, $\eta_{\mathrm{th}, \mathrm{wfl}}$ and $\eta_{\text {ex,wfl }}$. Specifically, $\eta_{\mathrm{th}, \mathrm{wfl}}$ showed a $5.1 \%$ variation over the investigated range of $C_{\mathrm{p}}$, and a $5.5 \%$ variation over the investigated range of $L_{\mathrm{p}}$. Similarly, over the same ranges $\eta_{\mathrm{ex}, \text { wfl }}$ varied by $68 \%$ and $75 \%$, respectively.

\subsection{Optimal Design of the Displacer Cylinder}

Equivalent plots to those in Fig. 3, but for the displacer cylinder are shown in Fig. 4. The capacitance and inductance of the displacer cylinder are given by $C_{\mathrm{d}}=\pi d_{\mathrm{d}}^{2} / 4 \rho g$ and $L_{\mathrm{d}}=4 \rho l_{\mathrm{d}} / \pi d_{\mathrm{d}}^{2}$, respectively, such that:

- An increase in the diameter of the displacer cylinder $d_{\mathrm{d}}$ causes $C_{\mathrm{d}}$ to increase and $L_{\mathrm{d}}$ to decrease;

- An increase in the liquid density of the working fluid $\rho$ causes $C_{\mathrm{d}}$ to decrease and $L_{\mathrm{d}}$ to increase; and,

- An increase in the time-averaged length of the liquid column in the displacer cylinder $l_{\mathrm{d}}$ (related to the time-average of level 7 in Fig. 2) causes $L_{\mathrm{d}}$ to increase, but would have no effect on $C_{\mathrm{d}}$.

From Fig. 4 it is observed that the magnitude of $\eta_{\text {dev }}$ is $3-6$ times smaller than that of $\eta_{\text {wfl }}$, or in absolute terms lower by $0.2-4.6 \%$. Moreover, the device efficiencies $\eta_{\text {dev }}$ are insensitive to the design of the displacer cylinder (i.e. the choice of $C_{\mathrm{d}}$ and $L_{\mathrm{d}}$ ), with $\eta_{\mathrm{th}, \mathrm{dev}} \sim 0.07 \%$ and $\eta_{\mathrm{ex}, \mathrm{dev}} \sim 0.9 \%$ (to within \pm 1 s.f.) over the entire investigated range. On the other hand $\eta_{\mathrm{wfl}}$ is approximately doubled by increasing $C_{\mathrm{d}}$ within the same range. Still, this doubling corresponds to a $0.2 \%$ absolute increase in $\eta_{\mathrm{th}, \mathrm{wfl}}$ and an $18 \%$ absolute increase in 
$\eta_{\text {ex,wfl }}$, which is considerably less than the improvement achievable with the careful design of the power cylinder suggested previously.

It is important to recognize that changes to the displacer cylinder parameters $\left(C_{\mathrm{d}}\right.$ and $\left.L_{\mathrm{d}}\right)$ can have contradictory results on the magnitude, but also the trends of the NIFTE's efficiency, depending on the choice of efficiency definition. This highlights the fact that the efficiency of the thermodynamic cycle undergone by the working fluid $\eta_{\mathrm{wfl}}$ can diverge significantly from the overall efficiency of the NIFTE device $\eta_{\mathrm{dev}}$, which includes the dissipation of useful power in the parasitic feedback valve that is external to the main thermodynamic cycle. By extension, it demonstrates that neglecting this dissipated power, as was assumed in Refs. [1-3] in order to allow a more simple analysis and interpretation of the NIFTE, is not generally valid. In this work we choose $\eta_{\mathrm{dev}}$ as a more representative indication of the overall capabilities of the NIFTE. On this basis, we will focus our discussion from this point onwards on the overall device efficiency $\eta_{\mathrm{dev}}$, though results will also be presented for the working fluid efficiency $\eta_{\mathrm{wfl}}$. We conclude that the displacer cylinder is not important in determining the efficiency of the NIFTE.

\subsection{Optimal Design of the Feedback Connection}

Plots concerning the feedback connection are shown in Fig. 5. The maximum achievable efficiency values obtained by making changes to the feedback connection variables are $\eta_{\mathrm{th}}=7.1 \%$ and $\eta_{\mathrm{ex}}=94 \%$. As with the power cylinder, these values are significantly higher than those obtained for the nominal NIFTE design (see Table 3), which again implies that the current design can be vastly improved with careful design. By comparison, typical thermal efficiencies of around 3 to $4 \%$ were reported for dry free-liquid-piston Stirling (Fluidyne) engines [8-11,22,23], though figures as high as $7 \%$ were reported for some larger units. The resistance and inductance of the feedback connection are given by $R_{\mathrm{f}}=128 \mu l_{\mathrm{f}} / \pi d_{\mathrm{f}}^{4}$ and $L_{\mathrm{f}}=4 \rho l_{\mathrm{f}} / \pi d_{\mathrm{f}}^{2}$, so:

- An increase in the length of the feedback connection $l_{\mathrm{f}}$ causes both $R_{\mathrm{f}}$ and $L_{\mathrm{f}}$ to increase;

- An increase in the diameter of the feedback connection $d_{\mathrm{f}}$ causes both $R_{\mathrm{f}}$ and $L_{\mathrm{f}}$ to decrease;

- An increase in the liquid dynamic viscosity of the working fluid $\mu$ causes $R_{\mathrm{f}}$ to increase, but has not effect on $L_{\mathrm{f}}$; and,

- An increase in the liquid density of the working fluid $\rho$ causes $L_{\mathrm{d}}$ to increase, but has no effect on $R_{\mathrm{f}}$.

The feedback connection, like the power cylinder, has an important role to play in determining the eventual efficiency of the NIFTE. An examination of the effects of the parameters $R_{\mathrm{f}}$ and $L_{\mathrm{f}}$ on our selected efficiency definition $\eta_{\mathrm{dev}}$ (i.e. only the circles) in Fig. 5, reveals that changes to $R_{\mathrm{f}}$ have a much greater ability to influence $\eta_{\mathrm{dev}}$ than changes to $L_{\mathrm{f}}$. In particular, Fig. 5 (left) shows that $R_{\mathrm{f}}$ (filled circles) can be used to improve $\eta_{\mathrm{th}, \mathrm{dev}}$ by up to $3.9 \%$ in absolute terms, and (right) to improve $\eta_{\mathrm{ex}, \mathrm{dev}}$ by up to $51 \%$ in absolute terms. The corresponding values for $L_{\mathrm{f}}$ (hollow circles) are $0.6 \%$ and $9 \%$, respectively. Thus, the sensitivities of $\eta_{\mathrm{th}, \mathrm{dev}}$ and $\eta_{\mathrm{ex}, \mathrm{dev}}$ to $R_{\mathrm{f}}$ are about 6 times greater than their sensitivities to $L_{\mathrm{f}}$. The sensitivities of the important device efficiency definitions $\eta_{\text {dev }}$ to all NIFTE parameters will be examined in detail in the discussion relating to Figs. 7 and 8.

So an efficiency improvement can be achieved by reducing $R_{\mathrm{f}}$, and to a lesser extent $L_{\mathrm{f}}$. Both of these can be realized simultaneously by decreasing the length $l_{\mathrm{f}}$ and increasing the diameter $d_{\mathrm{f}}$ of the feedback connection. An additional decrease in the liquid dynamic viscosity of the working fluid $\mu$ and a decrease in the liquid density of the working fluid $\rho$ will also act to independently lessen $R_{\mathrm{f}}$ and $L_{\mathrm{f}}$, respectively.

Recall however, from the sub-section on the power cylinder design, that an increased liquid density $\rho$ was required there for maximum efficiency. Hence, the optimization of the NIFTE with respect to this variable is more complex. One approach to avoid this conflict in requirements is to set $R_{\mathrm{f}}$ and $L_{\mathrm{f}}$ by designing the geometry of the feedback connection $\left(l_{\mathrm{f}}\right.$ and $d_{\mathrm{f}}$ ), and to set $C_{\mathrm{p}}$ and $L_{\mathrm{p}}$ independently by designing the geometry of the power cylinder $\left(d_{\mathrm{p}}\right)$, without the need to change the working fluid.

\subsection{Optimal Design of the Heat Exchangers and Adiabatic Vapor Chamber}

Finally, we turn to the heat exchangers and the total vapor volume at the top of the NIFTE. Plots concerning the effect of these two components on the efficiency of the NIFTE are shown in Fig. 6. The heat transfer process in the heat exchangers is described by the thermal resistance $R_{\mathrm{th}}=\left(\Delta s_{\mathrm{fg}} / \Delta v_{\mathrm{fg}}\right)^{2} T_{\mathrm{o}} / k$, while the adiabatic capacitance associated with the adiabatic vapor chamber is $C_{\mathrm{ad}}=V_{\mathrm{ao}} / \gamma P_{\mathrm{ao}}$. From these expressions, an increase in $R_{\text {th }}$ can be established with either a:

- Higher specific enthalpy due to phase change $\Delta s_{\mathrm{fg}}$; or, 
- Lower specific volume due to phase change $\Delta v_{\mathrm{fg}}$; or,

- Higher time-averaged (saturation) temperature $T_{0}$; or,

- Lower thermal conductance $k$.

In addition, $C_{\mathrm{ad}}$ can be increased with either a:

- Higher mean total volume in the vapor phase $V_{\mathrm{ao}}$; or,

- Higher ratio of heat capacities of the working fluid in the vapor phase $\gamma$; or,

- Lower time-averaged (saturation) pressure $P_{\mathrm{ao}}$, which is directly linked to the (saturation) temperature $T_{\mathrm{o}}$.

As before, we inspect the effects of the two independent variables (here $R_{\mathrm{th}}$ and $C_{\mathrm{ad}}$ ) on our selected (device) efficiency definition $\eta_{\mathrm{dev}}$ (i.e. only the circles) in Fig. 6 . The thermal resistance $R_{\text {th }}$ (filled circles) has a less noticeable effect on $\eta_{\text {dev }}$ than that of $C_{\text {ad }}$ (hollow circles). In fact, in Fig. 6 the sensitivities of both $\eta_{\mathrm{th}, \mathrm{dev}}$ and $\eta_{\mathrm{ex}, \mathrm{dev}}$ to $C_{\mathrm{ad}}$ are approximately 5 times greater than their sensitivities to $R_{\mathrm{th}}$, so the first priority in maximizing $\eta_{\mathrm{dev}}$ should be to maximize $C_{\mathrm{ad}}$. In more detail, decreasing $R_{\mathrm{th}}$ within the investigated range increases $\eta_{\mathrm{th} \text {,dev }}$ by $0.1 \%$ (or a factor of about 2), and increasing $C_{\mathrm{ad}}$ increases $\eta_{\mathrm{th}, \mathrm{dev}}$ by $0.5 \%$ (or a factor close to 5). Similarly, decreasing $R_{\mathrm{th}}$ increases $\eta_{\mathrm{ex}, \mathrm{dev}}$ by $1.4 \%$, and increasing $C_{\mathrm{ad}}$ increases $\eta_{\mathrm{ex}, \mathrm{dev}}$ by $6.9 \%$.

The selection of the working fluid is a complicated issue in itself, and depends on a number of additional factors (not contained in the current study) that must be weighed before a decision can be made, such as for example its boiling point in relation the hot $T_{\mathrm{h}}$ and cold $T_{\mathrm{c}}$ temperatures, amongst others. Specifically with respect to the boiling point, one basic requirement is that this must be somewhere between $T_{\mathrm{h}}$ and $T_{\mathrm{c}}$ if the working fluid is going to evaporate and condense, otherwise the NIFTE will not be able to operate.

Given that there is little scope in affecting ratio of heat capacities of the working fluid in the vapor phase $\gamma$, it is only possible to establish considerably larger values of $C_{\text {ad }}$ by increasing the total volume in the vapor phase $V_{\mathrm{ao}}$, and/or decreasing the time-averaged (saturation) pressure $P_{\mathrm{ao}}$. With respect to the former, the total adiabatic vapor chamber volume $V_{\text {ao }}$ can be increased by using power and displacer cylinders with larger diameters and lengths, as well as vapor connection pipes with larger diameters and lengths. Yet, the requirement concerning the power cylinder length is in partial contradiction with the condition that was established in the power cylinder sub-section for maximum efficiency, i.e. that a narrow (small $d_{\mathrm{p}}$ ) power cylinder ought to be preferred. One way to overcome this problem is to use a long, but small diameter power cylinder, thus achieving both small $C_{\mathrm{p}}$ and large $C_{\mathrm{ad}}$.

In contrast to $V_{\mathrm{ao}}$, the designer has little control over the time-averaged (saturation) pressure $P_{\mathrm{ao}}$. This pressure appears as an external boundary condition observed by the NIFTE, as it is determined by the time-mean pressure in the load to which the NIFTE is connected. Since it is coupled to the time-averaged temperature $T_{\mathrm{o}}$ that is found half-way between the source and sink temperatures, which also coincides with saturation conditions, $P_{\text {ao }}$ also determines the temperatures in the heat exchangers. In fact, we expect that lower $P_{\mathrm{a} o}$ will be associated with lower heat exchanger temperatures. Nevertheless, we expect that $C_{\mathrm{ad}}$ will be higher in these conditions, leading to improved efficiencies. A lower $T_{\mathrm{o}}$ will also help to decrease $R_{\mathrm{th}}$ that can have a secondary beneficial effect on the NIFTE efficiency. Note here however, that the linear analysis performed here is too simplistic to capture nonlinear effects that become increasingly important at higher $P_{\mathrm{ao}}$ and higher heat exchanger temperatures.

\subsection{Sensitivity Analysis}

In this section we re-examine the data presented previously, but in the form of a sensitivity analysis in order to identify the $R, C$ and $L$ parameters (and NIFTE variables) that most strongly affect the efficiencies of the NIFTE. We focus here only on the overall device efficiencies $\eta_{\mathrm{dev}}$, which include parasitic fluid power dissipation due to viscous and pressure drag in the feedback connection (due to $R_{\text {th }}$ ). Throughout this section we assume that the designer has no control over the load with which the device has to interface with, and hence set the load to its nominal value and exclude all load parameters from consideration.

Figure 7 (with key values summarized in Table 4) contains plots of the changes in the thermal efficiency of the device $\eta_{\text {th,dev }}$ due to changes in all independent device parameters, including: the capacitance due to hydrostatic pressure $C_{\mathrm{p}}$ and inductance due to liquid mass (inertia) in the power cylinder $L_{\mathrm{p}}$; the capacitance due to hydrostatic pressure $C_{\mathrm{d}}$ and inductance due to liquid mass (inertia) in the displacer cylinder $L_{\mathrm{d}}$; the resistance due to fluid flow (drag) $R_{\mathrm{f}}$ and inductance due to liquid mass (inertia) $L_{\mathrm{f}}$ in the feedback connection; the thermal resistance in the heat exchangers $R_{\mathrm{th}}$; and, the capacitance associated with compressibility of the adiabatic vapor chamber $C_{\mathrm{ad}}$. 
Figure 8 (with related key results summarized in Table 5) is identical to Fig. 7, but focuses on changes to the exergetic efficiency of the device $\eta_{\mathrm{ex}, \mathrm{dev}}$. It is possible to conclude from Figs. 7 and 8 , as well as the accompanying Tables 4 and 5, that the efficiencies of NIFTE are most sensitive to changes in the feedback connection (both $R_{\mathrm{f}}$ and $L_{\mathrm{f}}$ ), followed by the power cylinder $\left(C_{\mathrm{p}}\right.$ and $\left.L_{\mathrm{p}}\right)$, and to a lesser extent the thermal resistance in the heat exchangers $R_{\mathrm{th}}$ and the capacitance of the adiabatic vapor chamber $C_{\mathrm{ad}}$. Based on this information it becomes evident that the selection of the feedback valve as the natural operating point tuning component is the right approach.

\subsection{Temperature-Entropy Diagrams}

It has been shown in the previous sections that the feedback connection and power cylinder are important components that, if designed carefully, can lead to significant improvements in efficiency with respect to an existing (nominal) NIFTE prototype. For instance, a decrease in $R_{\mathrm{f}}$ resulted in an improvement in $\eta_{\mathrm{th}, \mathrm{dev}}$ from a nominal $0.1 \%$ up to $3.9 \%$ in absolute terms, and similarly in $\eta_{\text {ex,dev }}$ from a nominal $1 \%$ up to $52 \%$. In another example, a decrease in $C_{\mathrm{p}}$ was capable of increasing $\eta_{\mathrm{th} \text {,dev }}$ from a nominal $0.1 \%$ to $2.1 \%$, and $\eta_{\mathrm{ex} \text {,dev }}$ from a nominal $1 \%$ to $27 \%$. Corresponding working fluid efficiencies also showed considerable improvements, with $\eta_{\text {th,wfl }}$ increasing from $0.3 \%$ to $7.1 \%$ at the optimal $R_{\mathrm{f}}$ and $5.2 \%$ at the optimal $C_{\mathrm{p}}$, respectively; and with $\eta_{\text {ex,wfl }}$ increasing from $4 \%$ to $94 \%$ at the optimal $R_{\mathrm{f}}$ and $69 \%$ at the optimal $C_{\mathrm{p}}$ setting.

In order to gain some insight into the underlying reasons for these vast potential improvements, it is useful to examine the main (internally reversible) thermodynamic cycle undergone by the working fluid on a T-S diagram and also to compare this with the greater area that represents the full (thermally irreversible) cycle as experienced at the external heat source and sink. As explained previously, the distinction between these two rests on the choice of temperature, either $T$ or $T_{\mathrm{s}}$, and the area between the two represents a power loss (exergetic destruction) through irreversible heat transfer that occurs in $R_{\text {th }}$ between the source/sink and the working fluid. Figure 9 shows results from this investigation for the effects of $R_{\mathrm{f}}$ (left) and $C_{\mathrm{p}}$ (right) on the $T-S$ (solid lines, filled squares) and $T_{\mathrm{s}}-S$ (dotted lines, hollow squares) NIFTE diagrams undergone in the relative to the nominal configuration (circles).

The areas within the two ovals are a direct indication of the efficiencies of the corresponding cycle. As expected, it can be observed in Fig. 9 that the $T_{\mathrm{s}}-S$ diagrams (hollow symbols) always enclose the $T$-S diagrams (filled symbols). It can also be seen that the $T_{\mathrm{s}}-S$ diagrams cover the whole vertical range (height) in the graphs $T_{\mathrm{S}}{ }^{\prime}$ that corresponds to the amplitude of temperature oscillations in the heat exchangers $\widehat{T}_{\mathrm{s}}=15 \mathrm{~K}$, again as expected. The nominal design however has a greatly reduced working fluid temperature oscillation amplitude $\widehat{T}=1 \mathrm{~K}$, revealing a substantial exergetic loss in the thermal resistance $R_{\mathrm{th}}$. This helps to explain the relatively low $\eta_{\mathrm{ex}, \mathrm{wfl}}$ and $\eta_{\mathrm{ex}, \mathrm{dev}}$, since both of these efficiency measures are adversely affected by these exergetic losses.

Continuing, the most important observation that can be made based on the plots in Fig. 9 concerns the effects on the $T$-S diagrams (solid lines, square symbols) of optimizing either the value of $R_{\mathrm{f}}$ (left) or $C_{\mathrm{p}}$ (right). Compared to the nominal NIFTE, the optimized designs are associated with much larger amplitudes in the working fluid temperature oscillations. Specifically, we can see clearly from these plots that the underlying effect of the parametric optimization process is to increase $\hat{T}$ to $14 \mathrm{~K}$ when $R_{\mathrm{f}}$ is optimized and to $10 \mathrm{~K}$ when $C_{\mathrm{p}}$ is optimized. This corresponds directly to the higher $\eta_{\mathrm{ex}}$ in the optimal designs relative to the nominal NIFTE. In addition, the larger relative increase in $\widehat{T}$ that can be generated by varying $R_{\mathrm{f}}$ rather than $C_{\mathrm{p}}$ within their perturbation envelopes can explain the higher $\eta_{\text {ex }}$ of the $R_{\mathrm{f}}$ optimal NIFTE design. These values are summarized in Table 6.

As a final comment, it can be observed in Table 6 that unlike the nominal NIFTE, the optimized designs are capable of keeping the phase between $\Varangle\left\{T_{S}, \dot{S}\right\}$ and $\Varangle\{T, \dot{S}\}$ low. This phasing is an important parameter in determining the eventual efficiency as it controls (together with the amplitudes $\widehat{T}_{\mathrm{s}}$ and $\widehat{T}$ ) the area of the Lissajous ovals in Fig. 9, with zero phase corresponding to maximum efficiency and $90^{\circ}$ phase corresponding to zero efficiency. However, close inspection of various other sub-optimal designs (not shown here) reveals even lower phases than those reported in Table 6, suggesting that the primary reason for efficiency improvement at lower $R_{\mathrm{f}}$ and $C_{\mathrm{p}}$ is the increase in temperature amplitudes.

\section{CONCLUSIONS}

Starting from a first-order linear dynamic model of the NIFTE that consists of a network of interconnected spatially lumped components, we investigated parametrically the effect of various component variables (geometric and other) on the thermal (first law) and exergetic (second law) efficiencies of this device. Results from the 
parametric optimization process in which the parameters were varied independently within a specified range, were then compared to efficiency values associated with a nominal NIFTE configuration, based on values that were estimated from an existing prototype. Great improvements were demonstrated relative to the nominal NIFTE design, implying that the careful design can have a significant effect on the performance of the final device.

The study highlighted critical components that require careful design for the optimization of the NIFTE, namely the feedback connection valve, the power cylinder, the adiabatic volume and the thermal resistance in the heat exchangers. Relative to the nominal NIFTE, an efficient design would feature a lower feedback connection valve resistance, with a shorter connection length and larger connection diameter; a smaller diameter but taller power cylinder; a larger (time-mean) combined vapor volume at the top part of the device; as well as improved heat transfer behavior (i.e. reduced thermal resistance) in the hot and cold heat exchanger blocks.

The effect of these modifications was to increase the exergetic efficiency of the device by $50 \%$ points, which also corresponds to a $3.8 \%$ point increase in thermal efficiency. The NIFTE was modeled as operating across a temperature difference between heat source and sink that one would associate with low grade heat $(30 \mathrm{~K})$. Given a maximum theoretical (Carnot) efficiency associated with these temperatures of $9.5 \%$, the reported improvement is considered noteworthy. Close inspection of T-S diagrams revealed that the underlying reason for the improvement was an increase in the working fluid temperature amplitude as it undergoes the thermodynamic cycle.

\section{ACKNOWLEDGEMENTS}

The authors would like to thank Mr. Y. Malik and Miss R. Solanki for their assistance in generating the data.

\section{REFERENCES}

1. Smith TCB. Power dense thermofluidic oscillators for high load applications. In: Energy conversion engineering. IECEC 2004: Proceedings of the 2nd International Energy Conversion Engineering Conference; 2004 Aug 16-19; Providence (RI), USA. Reston (VA): AIAA; 2004. p. AIAA-2004-5758 1-15.

2. Smith TCB. Asymmetric heat transfer in vapour cycle liquid-piston engines. In: Stirling engine. ISEC 2005: Proceedings of the 12th International Stirling Engine Conference and Technology Exhibition; 2005 Sept 7-9; Durham, UK. Durham: Durham University; 2005. p. 302-14.

3. Smith TCB. Thermally driven oscillations in dynamic applications [ $\mathrm{PhD}$ thesis]. Cambridge: University of Cambridge, UK; 2006.

4. Backhaus S, Swift GW. A thermoacoustic-Stirling heat engine: Detailed study. J Acoust Soc Am 2000;107:3148-66.

5. Wheatley J, Hofler T, Swift GW, Migliori A. An intrinsically irreversible thermoacoustic heat engine. J Acoust Soc Am 1983;74:153-70.

6. Wheatley J, Hofler T, Swift GW, Migliori A. Experiments with an intrinsically irreversible acoustic heat engine. Phys Rev Lett 1983;50:499-502.

7. Ceperley PH. A pistonless Stirling engine-The travelling wave heat engine. J Acoust Soc Am 1979;66:1508-13.

8. West C. The Fluidyne Heat Engine. Research report. Harwell: Atomic Energy Research Establishment; 1971. Report No. AERE-R 6775.

9. West CD, Pandey RB. Laboratory prototype Fluidyne water pump. In: Energy conversion engineering. IECEC 1981: Proceedings of the 16th Intersociety Energy Conversion Engineering Conference; 1981 Aug 9-14; Atlanta (GA), USA. New York (NY): ASME; 1981. p. 1916-8.

10. West CD. Dynamic analysis of the Fluidyne. In: Energy conversion engineering. IECEC 1983: Proceedings of the 18th Intersociety Energy Conversion Engineering Conference; 1983 Aug 21-26; Orlando (FL), USA. New York (NY): AIChE; 1983. p. 779-84.

11. Stammers CW. The operation of the Fluidyne heat engine at low differential temperatures. J Sound Vib 1979;63:507-16.

12. Redlich RW, Berchowitz DM. Linear dynamics of free-piston Stirling engines. Proc Inst Mech Eng, Part A: Power Process Eng 1985;199:203-13.

13. Walker G, Senft JR. Free piston Stirling engines. Berlin: Springer; 1985.

14. Wood JG, Lane NW. Advanced 35 We Stirling engine for space power applications. In: El-Genk MS, editor. STAIF 2003: Proceedings of the Space Technology and Applications International Forum; 2003 Feb 2-5; Albuquerque (NM), USA. Melville (NY): AIP; 2003. p. 662-7.

15. Huang BJ, Chuang MD. System design of orifice pulse-tube refrigerator using linear flow network analysis. Cryog 1996;36:889-902. 
16. Payne PR, Brown RG, Brown JP. Water pulsejet research. Final report. Annapolis (MD): Payne Inc.; 1979 Apr. Report No. DOE/ET/20288-T1. Contract No.: AC01-77ET20288. Sponsored by the Department of Energy.

17. Kentfield JAC. Fundamentals of idealized airbreathing pulse-detonation engines. J Propuls Power 2002;18:78-83.

18. Kentfield JAC. Thermodynamics of airbreathing pulse-detonation engines. J Propuls Power 2002;18:1170-5.

19. Organ AJ. Stirling and pulse-tube cryo-coolers. 1st ed. Bury St. Edmunds: John Wiley and Sons; 2005.

20. Solanki R, Galindo A, Markides CN. Dynamic modelling of a two-phase thermofluidic oscillator for efficient low grade heat utilization: Effect of fluid inertia. J Appl Energy 2011; in press.

21. Ünsal B, Ray S, Durst F, Ertunç Ö. Pulsating laminar pipe flows with sinusoidal mass flux variations. Fluid Dyn Res 2005;37:317-33.

22. Goldberg LF, Rallis CJ. A prototype liquid-piston free-displacer Stirling engine. In: Energy conversion engineering. IECEC 1979: Proceedings of the 14th Intersociety Energy Conversion Engineering Conference; 1979 Aug 5-10; Boston (MA), USA. Washington (DC): ACS; 1979. p. 1103-8.

23. Elston MJ, Lurie MS, Rallis CJ, Kilgour DB. Further development of the Fluidyne liquid-piston engine. In: Energy conversion engineering. IECEC 1982: Proceedings of the 17th Intersociety Energy Conversion Engineering Conference; 1982 Aug 8-12; Los Angeles (CA), USA. New York (NY): IEEE; 1982. p. 1755-60. 


\section{TABLES}

TABLE 1. Expressions for the components appearing in the NIFTE as modeled in Fig. 2. The subscript 'fg' refers to phase change and 'o' to a time-averaged value; $\Delta s_{\mathrm{fg}}$ and $\Delta v_{\mathrm{fg}}$ denote the specific entropy and specific volume changes associated with phase change; $k$ is a relevant thermal conductance; $T_{\mathrm{o}}$ and $P_{\mathrm{o}}$ are the time-averaged temperature and pressure; $V_{\mathrm{ao}}$ is the mean total volume in the vapor phase; $\mu$ and $\rho$ are the viscosity and density of the liquids; and $l_{\mathrm{i}}$ and $d_{\mathrm{i}}$ are pipe lengths and diameters.

\begin{tabular}{lcc}
\hline Thermal-Fluid Effect & Electrical Component Analogy & Expression \\
\hline Heat exchanger thermal resistance & Resistor & $R_{\mathrm{th}}=\left(\Delta s_{\mathrm{fg}} / \Delta v_{\mathrm{fg}}\right)^{2} T_{\mathrm{o}} / k$ \\
Feedback valve \& Load flow drag (viscous/pressure) & Resistor & $R_{\mathrm{l}}=128 \mu l_{\mathrm{l}} / \pi d_{\mathrm{l}}^{4} \& R_{\mathrm{f}}=128 \mu l_{\mathrm{f}} / \pi d_{\mathrm{f}}^{4}$ \\
Power \& Displacer cylinder hydrostatic pressure & Capacitor & $C_{\mathrm{p}}=\pi d_{\mathrm{p}}^{2} / 4 \rho g \& C_{\mathrm{d}}=\pi d_{\mathrm{d}}^{2} / 4 \rho g$ \\
Adiabatic vapor chamber compressibility & Capacitor & $C_{\mathrm{ad}}=V_{\mathrm{ao}} / \gamma P_{\mathrm{ao}}$ \\
Power \& Displacer cylinder inertia (liquid mass) & Inductor & $L_{\mathrm{p}}=4 \rho l_{\mathrm{p}} / \pi d_{\mathrm{p}}^{2} \& L_{\mathrm{d}}=4 \rho l_{\mathrm{d}} / \pi d_{\mathrm{d}}^{2}$ \\
Feedback connection \& Load inertia (liquid mass) & Inductor & $L_{\mathrm{f}}=4 \rho l_{\mathrm{f}} / \pi d_{\mathrm{f}}^{2} \& L_{\mathrm{l}}=4 \rho l_{\mathrm{l}} / \pi d_{\mathrm{l}}^{2}$
\end{tabular}

TABLE 2. Investigated values of all electrical parameters in Fig. 2 and Table 1. Showing both nominal values that correspond to the actual NIFTE prototype described in detail in Ref. [3]; and the full investigated range of each parameter.

\begin{tabular}{|c|c|c|c|c|}
\hline \multirow[b]{2}{*}{ Electrical Parameter } & \multirow{2}{*}{$\begin{array}{c}\text { Symbol and } \\
\text { Definition }\end{array}$} & \multicolumn{2}{|r|}{ Value } & \multirow[b]{2}{*}{ Units } \\
\hline & & Nominal & Investigated Range & \\
\hline Power cylinder hydrostatic capacitance & $C_{\mathrm{p}}=\pi d_{\mathrm{p}}^{2} / 4 \rho g$ & $7.43 \times 10^{-8}$ & $3.22 \times 10^{-9}$ to $1.29 \times 10^{-6}$ & $\mathrm{~m}^{4} \mathrm{~s}^{2} / \mathrm{kg}$ \\
\hline Power cylinder liquid mass inductance & $L_{\mathrm{p}}=4 \rho l_{\mathrm{p}} / \pi d_{\mathrm{p}}^{2}$ & $3.77 \times 10^{5}$ & $7.90 \times 10^{3}$ to $3.16 \times 10^{7}$ & $\mathrm{~kg} / \mathrm{m}^{4}$ \\
\hline Displacer cylinder hydrostatic capacitance & $C_{\mathrm{d}}=\pi d_{\mathrm{d}}^{2} / 4 \rho g$ & $7.35 \times 10^{-8}$ & $1.59 \times 10^{-8}$ to $1.21 \times 10^{-6}$ & $\mathrm{~m}^{4} \mathrm{~s}^{2} / \mathrm{kg}$ \\
\hline Displacer cylinder liquid mass inductance & $L_{\mathrm{d}}=4 \rho l_{\mathrm{d}} / \pi d_{\mathrm{d}}^{2}$ & $1.80 \times 10^{5}$ & $8.50 \times 10^{3}$ to $6.40 \times 10^{6}$ & $\mathrm{~kg} / \mathrm{m}^{4}$ \\
\hline Feedback connection fluid flow resistance & $R_{\mathrm{f}}=128 \mu l_{\mathrm{f}} / \pi d_{\mathrm{f}}^{4}$ & $2.13 \times 10^{6}$ & $7.10 \times 10^{1}$ to $4.44 \times 10^{9}$ & $\mathrm{~kg} / \mathrm{m}^{4} \mathrm{~s}$ \\
\hline Feedback connection liquid mass inductance & $L_{\mathrm{f}}=4 \rho l_{\mathrm{f}} / \pi d_{\mathrm{f}}^{2}$ & $4.74 \times 10^{6}$ & $1.58 \times 10^{4}$ to $3.95 \times 10^{8}$ & $\mathrm{~kg} / \mathrm{m}^{4}$ \\
\hline Heat exchanger thermal resistance & $R_{\mathrm{th}}=\left(\Delta h_{\mathrm{fg}} / \Delta v_{\mathrm{fg}}\right)^{2} / k T_{\mathrm{o}}$ & $5.02 \times 10^{8}$ & $3.27 \times 10^{7}$ to $6.74 \times 10^{9}$ & $\mathrm{~kg} / \mathrm{m}^{4} \mathrm{~s}$ \\
\hline Adiabatic vapor chamber compressibility & $C_{\mathrm{ad}}=V_{\mathrm{ao}} / \gamma P_{\mathrm{ao}}$ & $1.76 \times 10^{-9}$ & $1.06 \times 10^{-10}$ to $1.46 \times 10^{-7}$ & $\mathrm{~m}^{4} \mathrm{~s}^{2} / \mathrm{kg}$ \\
\hline Load fluid flow resistance & $R_{1}=128 \mu l_{1} / \pi d_{1}^{4}$ & $4.08 \times 10^{6}$ & $4.08 \times 10^{0}$ to $1.51 \times 10^{10}$ & $\mathrm{~kg} / \mathrm{m}^{4} \mathrm{~s}$ \\
\hline Load liquid mass inductance & $L_{1}=4 \rho l_{1} / \pi d_{1}^{2}$ & $1.27 \times 10^{7}$ & $1.27 \times 10^{3}$ to $4.24 \times 10^{9}$ & $\mathrm{~kg} / \mathrm{m}^{4}$ \\
\hline
\end{tabular}

TABLE 3. Nominal device and working fluid exergetic and thermal efficiencies corresponding to the actual NIFTE prototype.

\begin{tabular}{cc}
\hline Efficiency Definition & Value $(\%)$ \\
\hline$\eta_{\text {th,dev }}$ & 0.1 \\
$\eta_{\text {th,wfl }}$ & 0.3 \\
$\eta_{\text {ex,dev }}$ & 1.0 \\
$\eta_{\text {ex,wfl }}$ & 3.9
\end{tabular}

TABLE 4. Device thermal efficiency $\eta_{\text {th,dev }}$ sensitivity coefficients corresponding to Fig. 7. Showing both nominal coefficients that correspond to the actual NIFTE prototype described in detail in Ref. [3]; and the maximum (of the modulus) of each coefficient over the full investigated range of each parameter, as presented in Table 2.

\begin{tabular}{lccc}
\hline & Symbol and & Device Efficiency Sensitivity Coefficient \\
\cline { 2 - 4 } Parameter & Definition & Nominal & Maximum (Modulus) \\
\hline Power cylinder hydrostatic capacitance & $C_{\mathrm{p}}=\pi d_{\mathrm{p}}^{2} / 4 \rho g$ & $2.92 \times 10^{-3}$ & $1.85 \times 10^{-1}\left[{ }^{\mathrm{c}}\right]$ \\
Power cylinder liquid mass inductance & $L_{\mathrm{p}}=4 \rho l_{\mathrm{p}} / \pi d_{\mathrm{p}}^{2}$ & $3.05 \times 10^{-4}$ & $2.26 \times 10^{-3}$ \\
Displacer cylinder hydrostatic capacitance & $C_{\mathrm{d}}=\pi d_{\mathrm{d}}^{2} / 4 \rho g$ & $4.06 \times 10^{-4}$ & $4.21 \times 10^{-4}$ \\
Displacer cylinder liquid mass inductance & $L_{\mathrm{d}}=4 \rho l_{\mathrm{d}} / \pi d_{\mathrm{d}}^{2}$ & $2.66 \times 10^{-5}$ & $4.00 \times 10^{-5}$ \\
Feedback connection fluid flow resistance & $R_{\mathrm{f}}=128 \mu l_{\mathrm{f}} / \pi d_{\mathrm{f}}^{4}$ & $1.70 \times 10^{-3}$ & $1.45 \times 10^{+1}\left[{ }^{\mathrm{a}}\right]$ \\
Feedback connection liquid mass inductance & $L_{\mathrm{f}}=4 \rho l_{\mathrm{f}} / \pi d_{\mathrm{f}}^{2}$ & $7.41 \times 10^{-4}$ & $1.45 \times 10^{-1}\left[{ }^{\mathrm{b}}\right]$ \\
Heat exchanger thermal resistance & $R_{\mathrm{th}}=\left(\Delta s_{\mathrm{fg}} / \Delta v_{\mathrm{fg}}\right)^{2} T_{\mathrm{o}} / k$ & $2.63 \times 10^{-3}$ & $3.46 \times 10^{-3}$ \\
Adiabatic vapor chamber compressibility & $C_{\mathrm{ad}}=V_{\mathrm{ao}} / \gamma P_{\mathrm{ao}}$ & $9.65 \times 10^{-4}$ & $3.32 \times 10^{-3}$
\end{tabular}

${ }^{\mathrm{b}}$ Primary control component minor variable

${ }^{\mathrm{c}}$ Secondary control component main variable 
TABLE 5. Device exergetic efficiency $\eta_{\text {ex,dev }}$ sensitivity coefficients corresponding to Fig. 8 . Showing both nominal coefficients that correspond to the actual NIFTE prototype described in detail in Ref. [3]; and the maximum (of the modulus) of each coefficient over the full investigated range of each parameter, as presented in Table 2.

\begin{tabular}{lccc}
\hline & Symbol and & \multicolumn{2}{c}{ Sensitivity Coefficient } \\
\cline { 2 - 4 } Parameter & Definition & \multicolumn{2}{c}{ Nominal } \\
\hline Power cylinder hydrostatic capacitance & $C_{\mathrm{p}}=\pi d_{\mathrm{p}}^{2} / 4 \rho g$ & $1.19 \times 10^{-3}$ & $1.91 \times 10^{0}\left[{ }^{\mathrm{c}}\right]$ \\
Power cylinder liquid mass inductance & $L_{\mathrm{p}}=4 \rho l_{\mathrm{p}} / \pi d_{\mathrm{p}}^{2}$ & $1.05 \times 10^{-4}$ & $2.79 \times 10^{-2}$ \\
Displacer cylinder hydrostatic capacitance & $C_{\mathrm{d}}=\pi d_{\mathrm{d}}^{2} / 4 \rho g$ & $1.46 \times 10^{-3}$ & $3.41 \times 10^{-3}$ \\
Displacer cylinder liquid mass inductance & $L_{\mathrm{d}}=4 \rho l_{\mathrm{d}} / \pi d_{\mathrm{d}}^{2}$ & $1.68 \times 10^{-4}$ & $2.47 \times 10^{-4}$ \\
Feedback connection fluid flow resistance & $R_{\mathrm{f}}=128 \mu l_{\mathrm{f}} / \pi d_{\mathrm{f}}^{4}$ & $2.02 \times 10^{-4}$ & $1.93 \times 10^{+2}\left[{ }^{\mathrm{a}}\right]$ \\
Feedback connection liquid mass inductance & $L_{\mathrm{f}}=4 \rho l_{\mathrm{f}} / \pi d_{\mathrm{f}}^{2}$ & $3.97 \times 10^{-3}$ & $1.66 \times 10^{0}\left[{ }^{\mathrm{b}}\right]$ \\
Heat exchanger thermal resistance & $R_{\mathrm{th}}=\left(\Delta s_{\mathrm{fg}} / \Delta v_{\mathrm{fg}}\right)^{2} T_{\mathrm{o}} / k$ & $8.89 \times 10^{-3}$ & $2.38 \times 10^{-2}$ \\
Adiabatic vapor chamber compressibility & $C_{\mathrm{ad}}=V_{\mathrm{ao}} / \gamma P_{\mathrm{ao}}$ & $1.97 \times 10^{-3}$ & $9.26 \times 10^{-3}$
\end{tabular}

${ }^{\text {a }}$ Primary control component major variable

${ }^{\mathrm{b}}$ Primary control component minor variable

${ }^{\mathrm{c}}$ Secondary control component main variable

TABLE 6. Result of optimization of the NIFTE with respect to (w.r.t.) $R_{\mathrm{f}}$ and $C_{\mathrm{p}}$ in terms of external and working fluid temperature amplitudes $\left(\widehat{T}_{\mathrm{s}}\right.$ and $\left.\widehat{T}\right)$ and corresponding phases $\left(\Varangle\left\{T_{\mathrm{s}}, \dot{S}\right\}\right.$ and $\left.\Varangle\{T, \dot{S}\}\right)$. Comparison with the nominal design.

\begin{tabular}{cccc}
\hline & & \multicolumn{2}{c}{ Optimized NIFTE } \\
\cline { 3 - 4 } Variable & Nominal Value & w.r.t. $\boldsymbol{R}_{\mathbf{f}}$ & w.r.t. $\boldsymbol{C}_{\mathbf{p}}$ \\
\hline$\hat{T}_{\mathrm{s}}(\mathrm{K})$ & 15 & 15 & 15 \\
$\hat{T}(\mathrm{~K})$ & 1 & 14 & 10 \\
$\Varangle\left\{T_{\mathrm{s}}, \dot{S}\right\}$ (deg.) & $3^{\circ}$ & $8^{\circ}$ & $4^{\circ}$ \\
$\measuredangle\{T, \dot{S}\}$ (deg.) & $52^{\circ}$ & $8^{\circ}$ & $6^{\circ}$
\end{tabular}




\section{FIGURES}

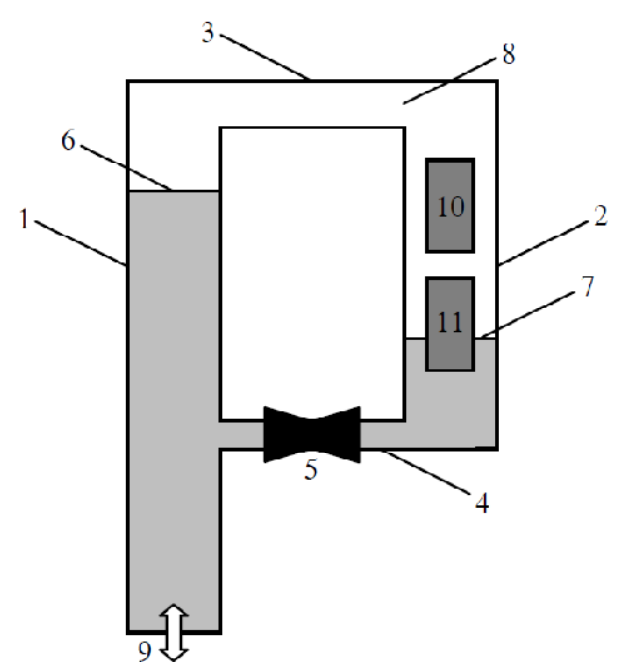

FIGURE 1. Schematic of the NIFTE. The area in white corresponds to the vapor volume in which the working fluid is in the vapor phase, while the area in gray corresponds to the liquid volume in which the working fluid is in the liquid phase.

Component 1 is the power cylinder (denoted by subscript ' $p$ ' in the text) and 2 is the displacer cylinder ('d'), 3 is the vapor connection, and, 4 and 5 are the liquid feedback connection and valve ('f'). Levels 6 and 7 are the working fluid vapor-liquid interfaces in the power (left) and displacer (right) cylinders. The combined vapor volume 8 (above levels 6 and 7) is assumed to be an adiabatic vapor chamber ('ad'). Interface 9 is connected to the load line ('l'). Components 10 (hot) and 11 (cold) are heat exchanger blocks ('th').

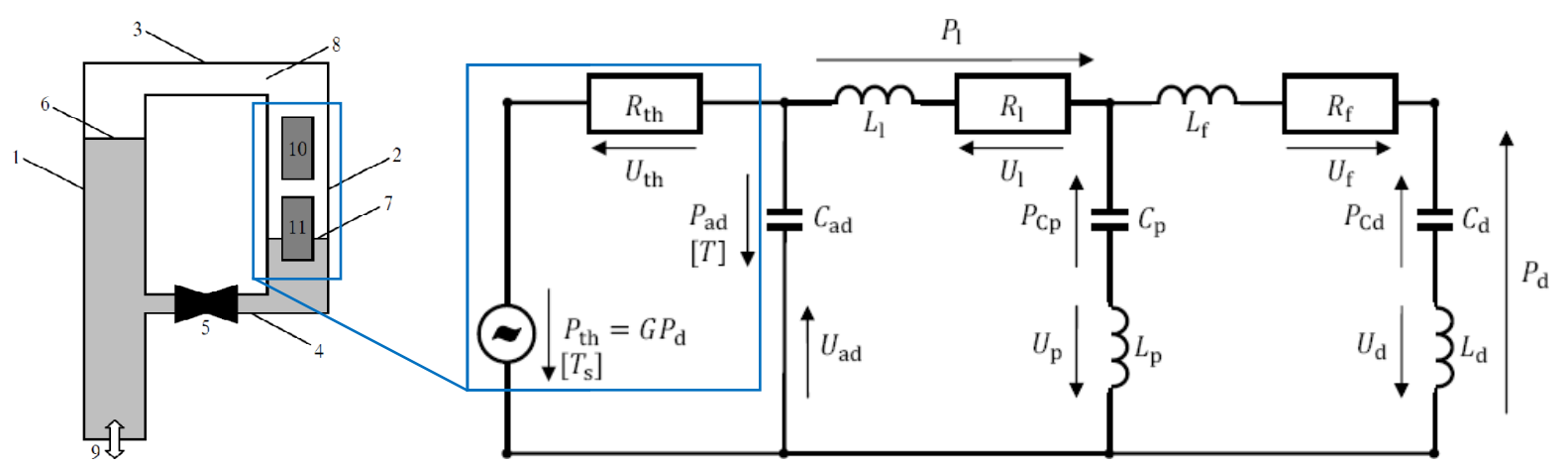

FIGURE 2. NIFTE model circuit diagram, where is the feedback gain, is a resistance, is a capacitance, is an inductance, is a pressure and is a volumetric flow rate. Subscript 'th' denotes the thermal domain (heat exchanger blocks 10 and 11 in the repeat of Fig. 1 on the left), 'ad' the adiabatic domain (vapor volume 8, above liquid levels 6 and 7), ' 1 ' the load (below 9), 'p' and 'd' the power (1) and displacer (2) cylinders, and ' $\mathrm{f}$ ' the feedback line and valve $(4,5)$. 

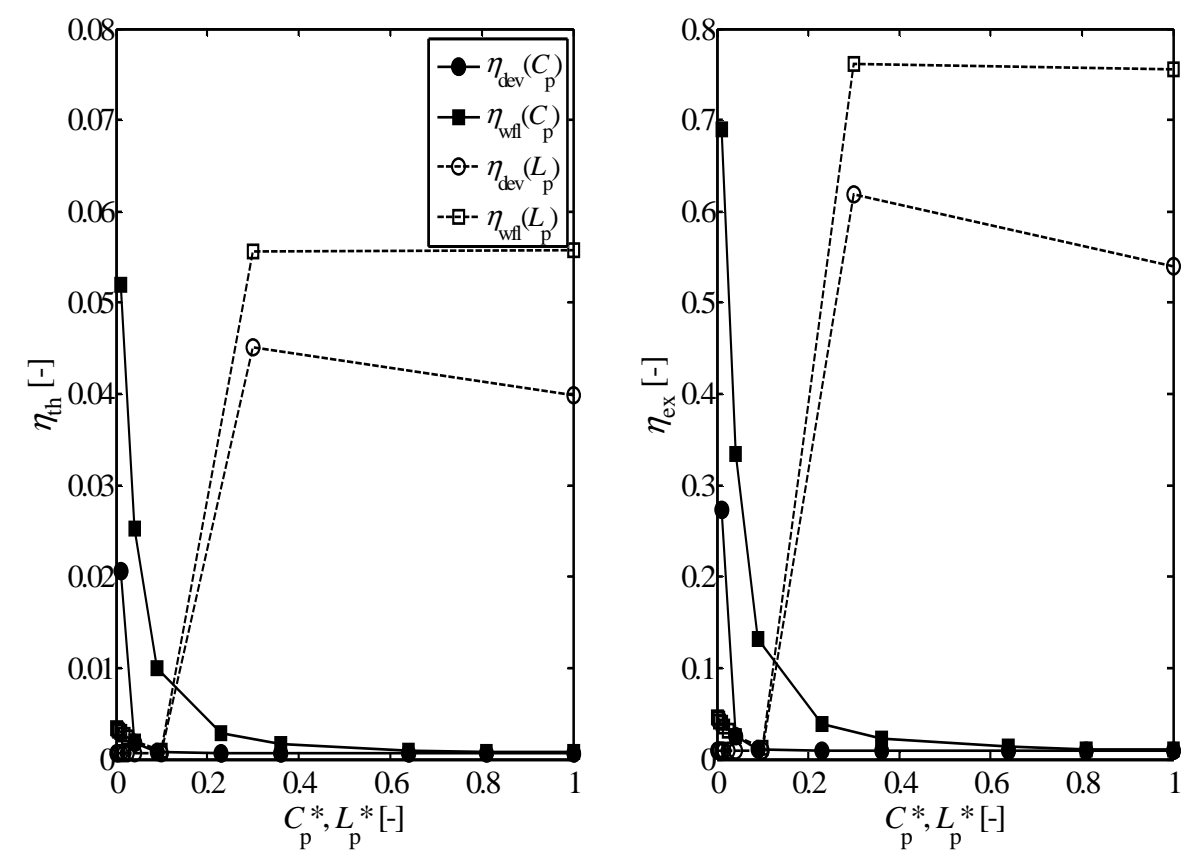

FIGURE 3. Effect of power cylinder capacitance $C_{\mathrm{p}}$ and inductance $L_{\mathrm{p}}$ on the NIFTE device and working fluid thermal efficiencies, $\eta_{\text {th,dev }}$ and $\eta_{\text {th,wfl }}$ respectively (left); and device and working fluid exergetic efficiencies, $\eta_{\text {ex,dev }}$ and $\eta_{\text {ex,wfl }}$ respectively (right). The investigated parameters were varied one at a time, with all others set to a nominal value. Abscissas normalized by the largest value in the investigated range.
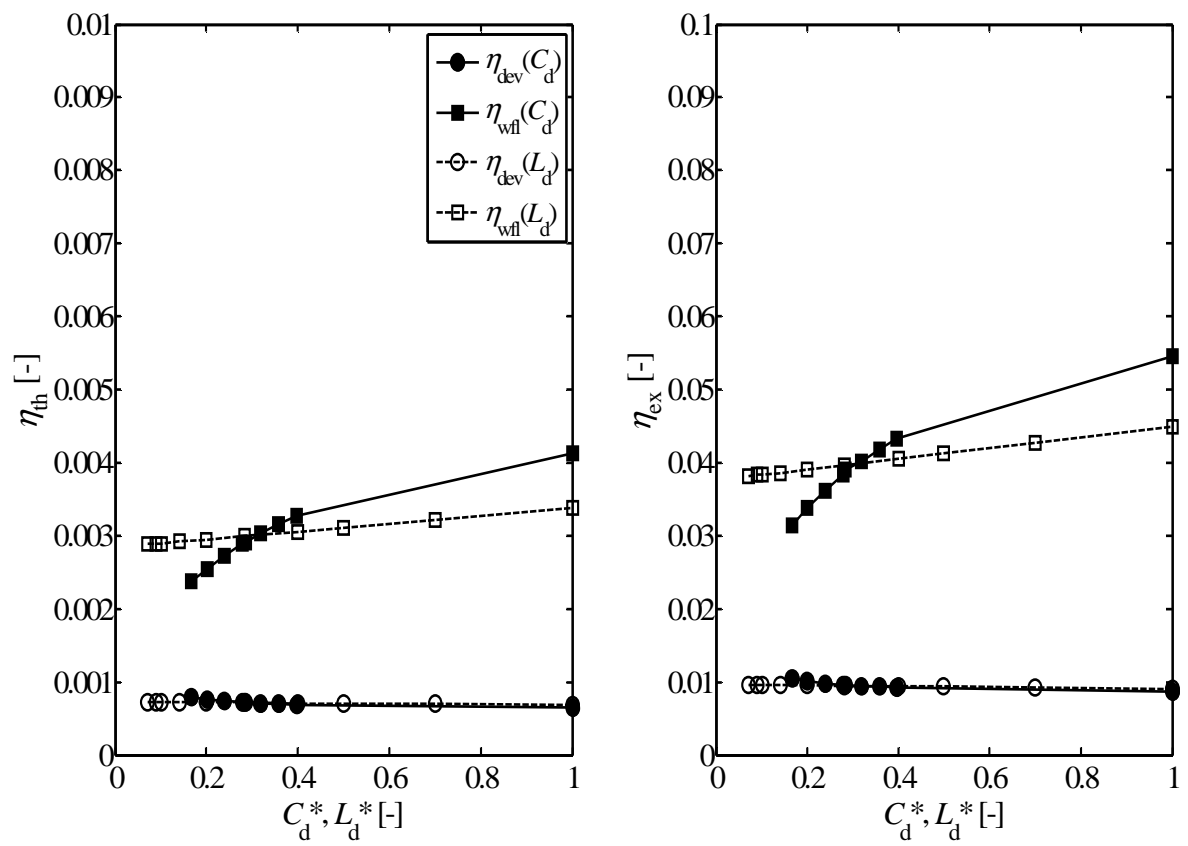

FIGURE 4. Effect of displacer cylinder capacitance $C_{\mathrm{d}}$ and inductance $L_{\mathrm{d}}$ on the NIFTE device and working fluid thermal efficiencies, $\eta_{\mathrm{th}, \mathrm{dev}}$ and $\eta_{\mathrm{th} \text {,wfl }}$ respectively (left); and device and working fluid exergetic efficiencies, $\eta_{\mathrm{ex}, \mathrm{dev}}$ and $\eta_{\mathrm{ex}, \mathrm{wfl}}$ respectively (right). The investigated parameters were varied one at a time, with all others set to a nominal value. Abscissas normalized by the largest value in the investigated range. 

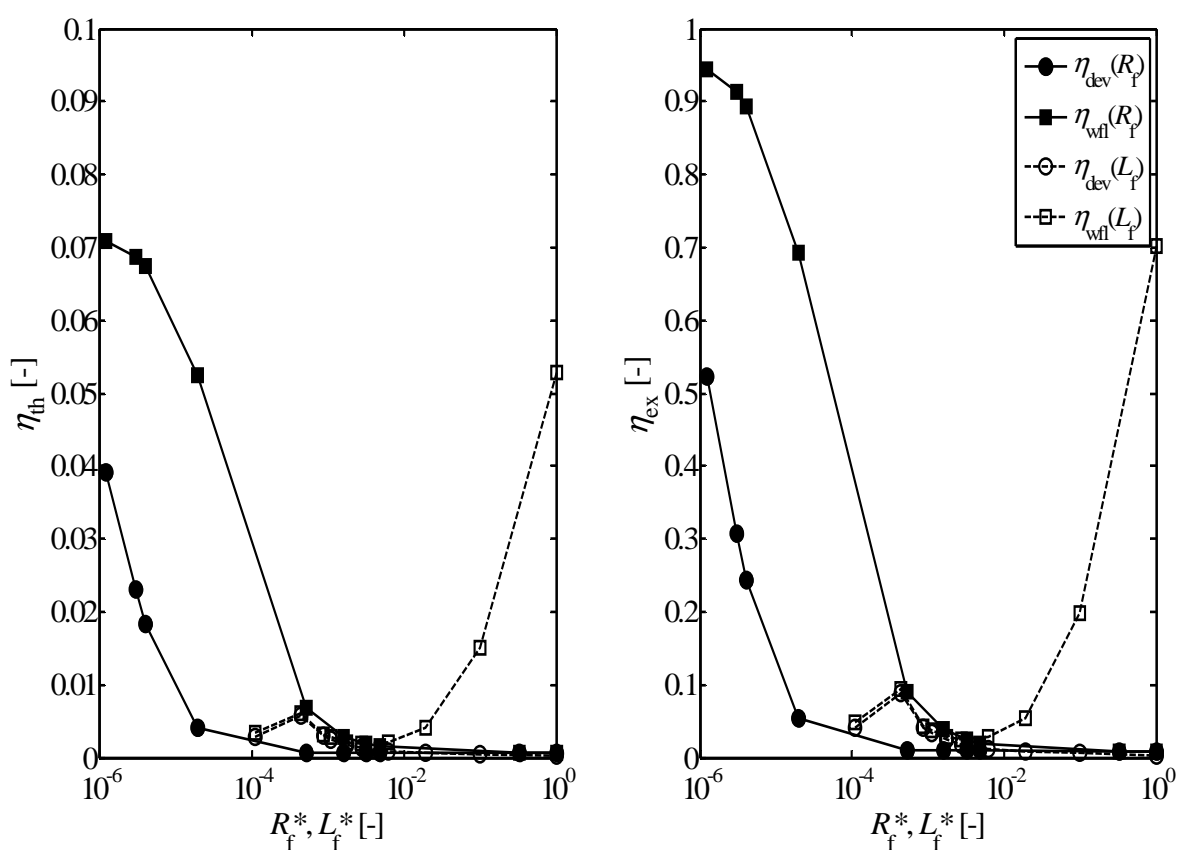

FIGURE 5. Effect of feedback valve resistance $R_{\mathrm{f}}$ and connection inductance $L_{\mathrm{f}}$ on the NIFTE device and working fluid thermal efficiencies, $\eta_{\mathrm{th}, \mathrm{dev}}$ and $\eta_{\mathrm{th}, \mathrm{wfl}}$ respectively (left); and device and working fluid exergetic efficiencies, $\eta_{\mathrm{ex}, \mathrm{dev}}$ and $\eta_{\text {ex,wfl }}$ respectively (right). The investigated parameters were varied one at a time, with all others set to a nominal value. Abscissas normalized by the largest value in the investigated range.
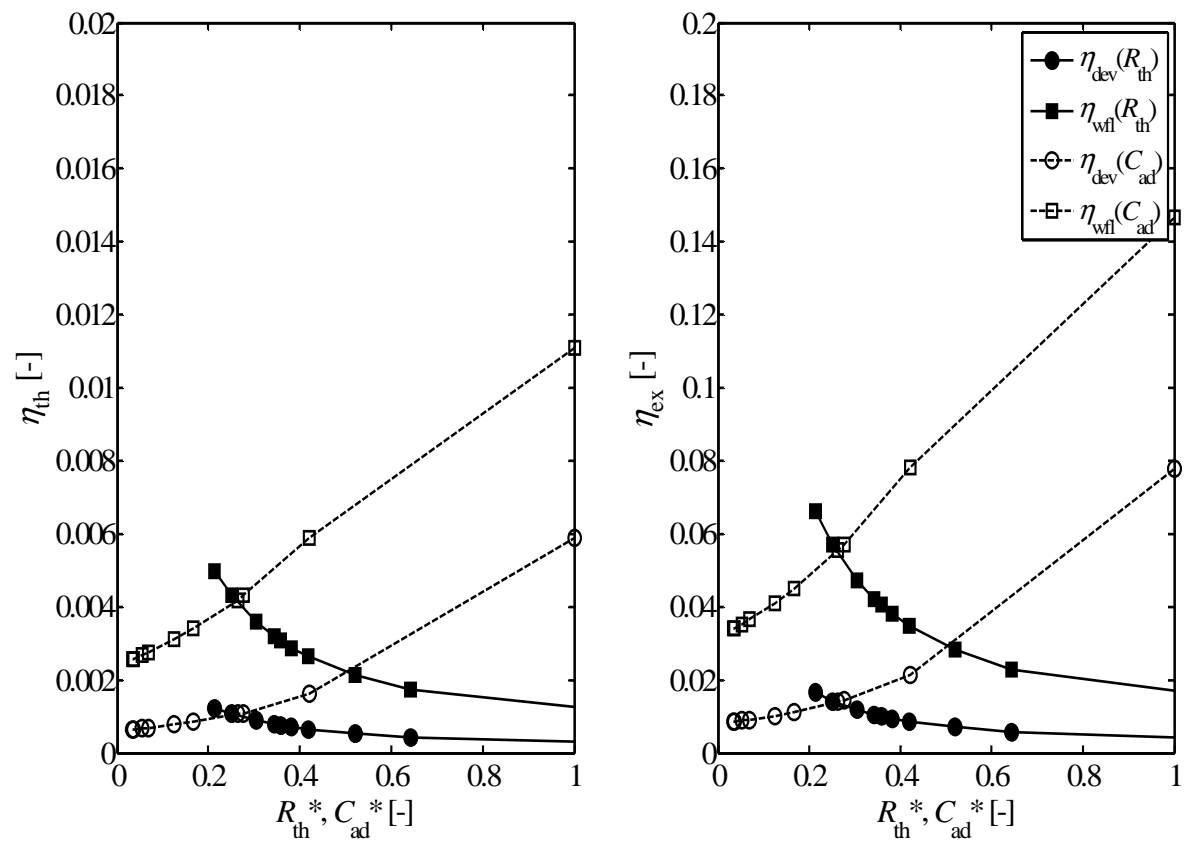

FIGURE 6. Effect of thermal resistance $R_{\text {th }}$ and adiabatic capacitance $C_{\text {ad }}$ on the NIFTE device and working fluid thermal efficiencies, $\eta_{\text {th,dev }}$ and $\eta_{\mathrm{th}, \mathrm{wfl}}$ respectively (left); and device and working fluid exergetic efficiencies, $\eta_{\mathrm{ex}, \mathrm{dev}}$ and $\eta_{\mathrm{ex}, \mathrm{wfl}}$ respectively (right). The investigated parameters were varied one at a time, with all others set to a nominal value. Abscissas normalized by the largest value in the investigated range. 


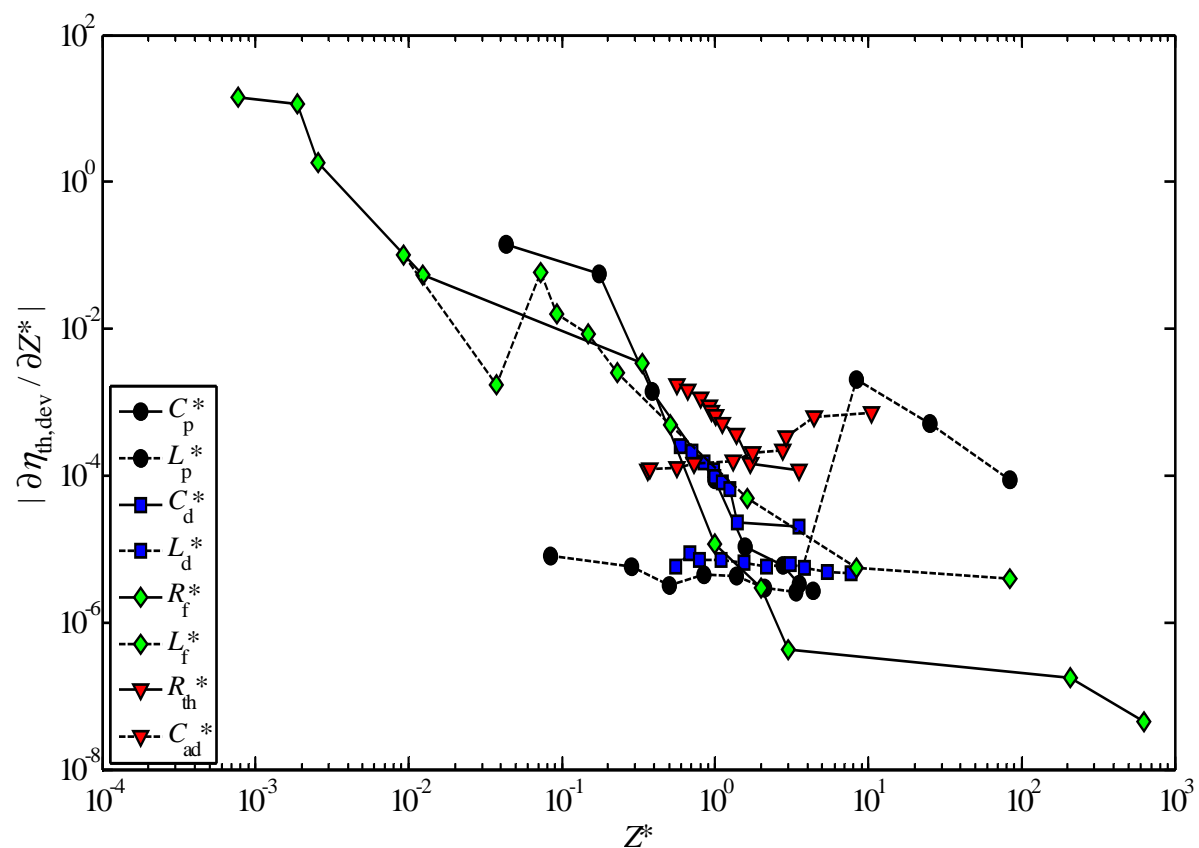

FIGURE 7. Change in the NIFTE device thermal efficiency $\eta_{\mathrm{th}, \mathrm{dev}}$ due to change in: hydrostatic pressure capacitance $C_{\mathrm{p}}$ and liquid mass inductance in the power cylinder $L_{\mathrm{p}}$; hydrostatic pressure capacitance $C_{\mathrm{d}}$ and liquid mass inductance in the displacer cylinder $L_{\mathrm{d}}$; fluid flow resistance $R_{\mathrm{f}}$ and liquid mass inductance $L_{\mathrm{f}}$ in the feedback connection; thermal resistance in the heat exchangers $R_{\mathrm{th}}$; and, adiabatic vapor chamber capacitance $C_{\mathrm{ad}}$. Abscissa values of impedance $Z^{*}$ on the horizontal axes have been normalized by each variable's nominal value.

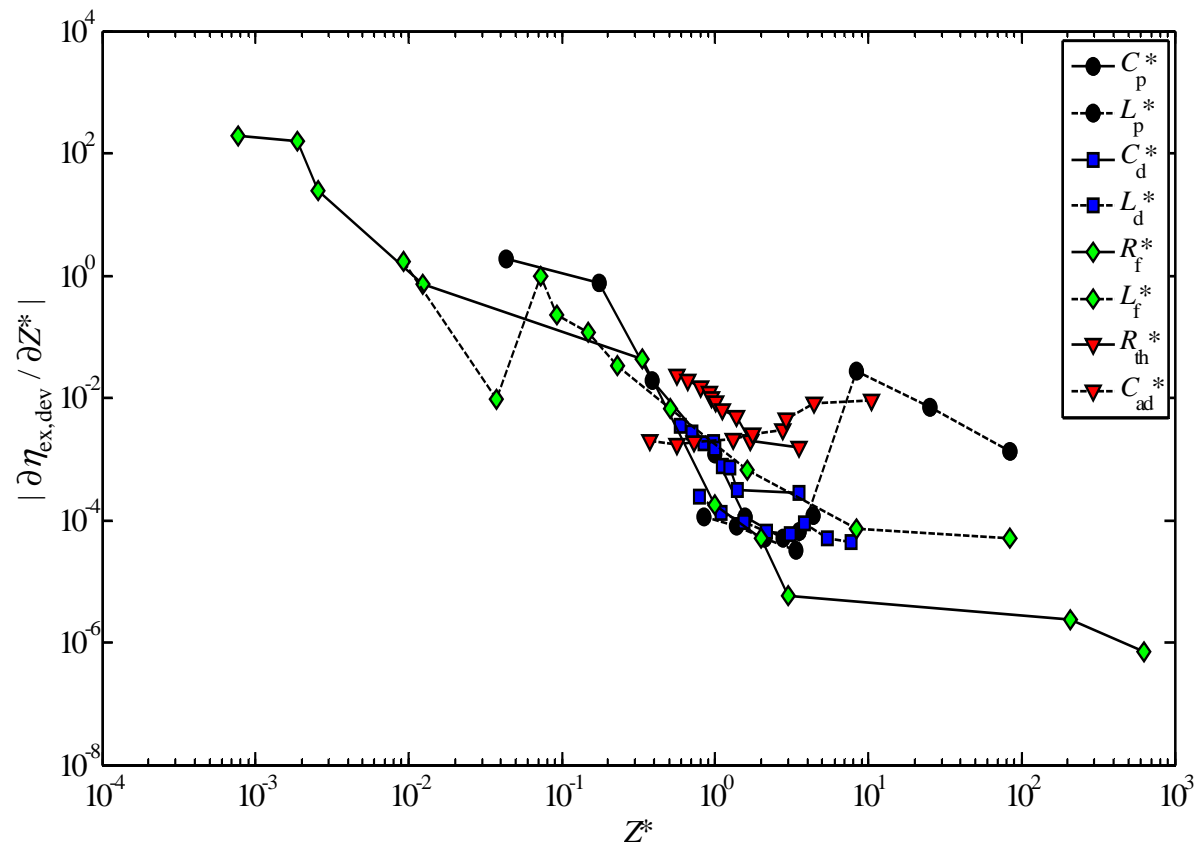

FIGURE 8. Change in the NIFTE device exergetic efficiency $\eta_{\text {ex,dev }}$ due to change in: hydrostatic pressure capacitance $C_{\mathrm{p}}$ and liquid mass inductance in the power cylinder $L_{\mathrm{p}}$; hydrostatic pressure capacitance $C_{\mathrm{d}}$ and liquid mass inductance in the displacer cylinder $L_{\mathrm{d}}$; fluid flow resistance $R_{\mathrm{f}}$ and liquid mass inductance $L_{\mathrm{f}}$ in the feedback connection; thermal resistance in the heat exchangers $R_{\mathrm{th}}$; and, adiabatic vapor chamber capacitance $C_{\mathrm{ad}}$. Abscissa values of impedance $Z^{*}$ on the horizontal axes have been normalized by each variable's nominal value. 

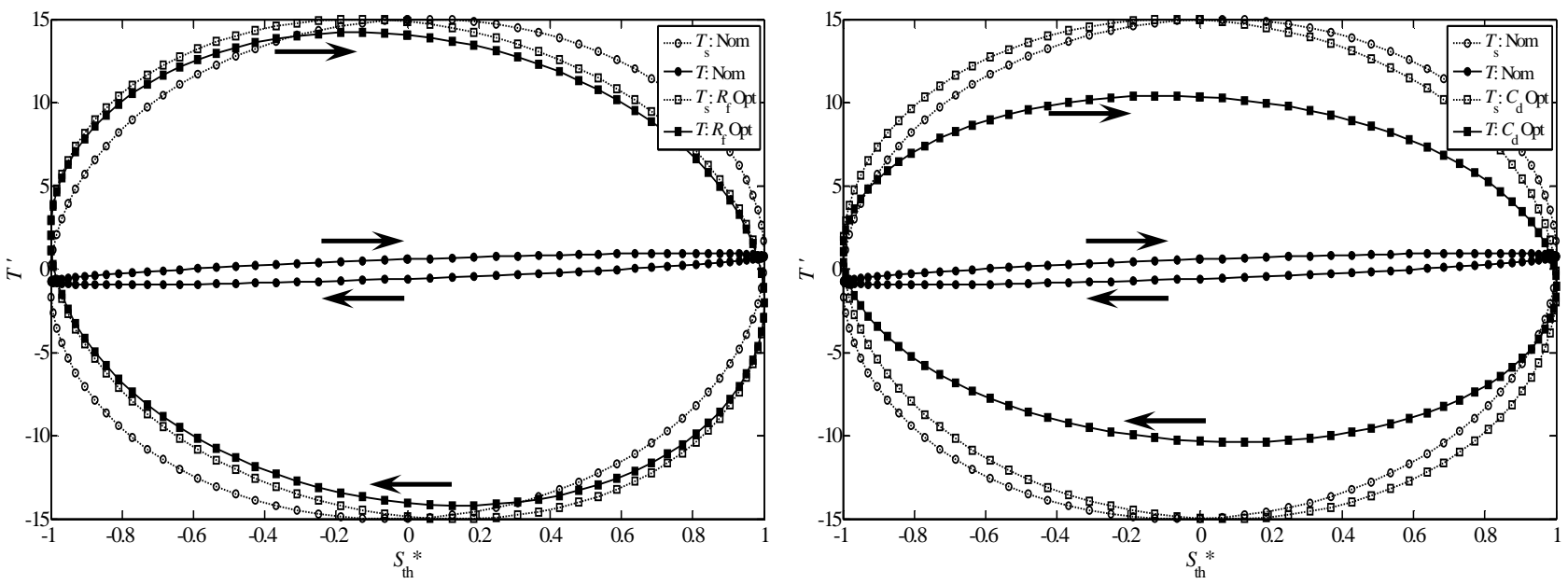

FIGURE 9. First order linear approximation phase plane plots (Lissajous ovals) of the NIFTE thermodynamic cycle, showing the nominal configuration (circles) with $R_{\mathrm{f}}=2.13 \times 10^{6}$ and $C_{\mathrm{p}}=7.43 \times 10^{-8}$, as well as optimized cycles with respect to $R_{\mathrm{f}}=1.64 \times 10^{3}$ (left, square symbols) and $C_{\mathrm{p}}=3.22 \times 10^{-9}$ (right, square symbols). Each figure includes both working fluid $T$-S (solid lines, filled symbols) and device $T_{\mathrm{s}}-S$ (dotted lines, hollow symbols) diagrams. Abscissa values of entropy fluctuations $S_{\mathrm{th}}{ }^{*}$ on the horizontal axes are normalized by their amplitude. 